\title{
Deep Solar Activity Minimum 2007 - 2009: Solar Wind Properties and Major Effects on the Terrestrial Magnetosphere
}

\author{
C.J. Farrugia · B. Harris • M. Leitner • C. Möstl • A.B. Galvin • K.D.C. Simunac • \\ R.B. Torbert • M.B. Temmer • A.M. Veronig • N.V. Erkaev • A. Szabo • K.W. Ogilvie • \\ J.G. Luhmann • V.A. Osherovich
}

Received: 4 November 2011 / Accepted: 13 August 2012 / Published online: 28 September 2012

(C) Springer Science+Business Media B.V. 2012

\begin{abstract}
We discuss the temporal variations and frequency distributions of solar wind and interplanetary magnetic field parameters during the solar minimum of 2007-2009 from measurements returned by the IMPACT and PLASTIC instruments on STEREO-A. We find that the density and total field strength were significantly weaker than in the previous minimum. The Alfvén Mach number was higher than typical. This reflects the weakness of magnetohydrodynamic (MHD) forces, and has a direct effect on the solar wind-magnetosphere interactions. We then discuss two major aspects that this weak solar activity had on the magnetosphere, using data from Wind and ground-based observations: i) the dayside contribu-
\end{abstract}

The Sun 360

Guest Editors: Bernhard Fleck, Bernd Heber, and Angelos Vourlidas

C.J. Farrugia $(\varangle)$ · B. Harris · M. Leitner · A.B. Galvin · K.D.C. Simunac · R.B. Torbert Space Science Center and Department of Physics, University of New Hampshire, Durham, NH, USA

e-mail: charlie.farrugia@unh.edu

M. Leitner

Institute for Astro- and Particle Physics, University of Innsbruck, Innsbruck 6020, Austria

C. Möstl · M.B. Temmer · A.M. Veronig

Kanzelhöhe Observatory - IGAM, Institute of Physics, University of Graz, Graz 8010, Austria

C. Möstl · M.B. Temmer

Space Research Institute, Austrian Academy of Sciences, Graz 8042, Austria

N.V. Erkaev

Institute for Computational Modeling, Russian Academy of Sciences, Krasnoyarsk, Russia

N.V. Erkaev

Siberian Federal University, Krasnoyarsk, Russia

A. Szabo - K.W. Ogilvie · V.A. Osherovich

NASA/Goddard Space Flight Center, Greenbelt, MD, USA

C. Möstl · J.G. Luhmann

Space Sciences Laboratory, Berkeley, CA, USA 
tion to the cross-polar cap potential (CPCP), and ii) the shapes of the magnetopause and bow shock. For i) we find a low interplanetary electric field of $1.3 \pm 0.9 \mathrm{mV} \mathrm{m}^{-1}$ and a CPCP of $37.3 \pm 20.2 \mathrm{kV}$. The auroral activity is closely correlated to the prevalent stream-stream interactions. We suggest that the Alfvén wave trains in the fast streams and Kelvin-Helmholtz instability were the predominant agents mediating the transfer of solar wind momentum and energy to the magnetosphere during this three-year period. For ii) we determine 328 magnetopause and 271 bow shock crossings made by Geotail, Cluster 1, and the THEMIS B and $\mathrm{C}$ spacecraft during a three-month interval when the daily averages of the magnetic and kinetic energy densities attained their lowest value during the three years under survey. We use the same numerical approach as in Fairfield's (J. Geophys. Res. 76, 7600, 1971) empirical model and compare our findings with three magnetopause models. The stand-off distance of the subsolar magnetopause and bow shock were $11.8 R_{\mathrm{E}}$ and $14.35 R_{\mathrm{E}}$, respectively. When comparing with Fairfield's (1971) classic result, we find that the subsolar magnetosheath is thinner by $\sim 1 R_{\mathrm{E}}$. This is mainly due to the low dynamic pressure which results in a sunward shift of the magnetopause. The magnetopause is more flared than in Fairfield's model. By contrast the bow shock is less flared, and the latter is the result of weaker MHD forces.

\section{Introduction}

The exceptionally long and pronounced minimum in solar activity at the transition from Solar Cycles 23 to 24, lasting from 2007 to 2009 inclusive, has excited a lot of attention in the community (see, for example, the compilation of articles in Cranmer, Hoeksema, and Kohl, 2010). Comparisons and contrasts have been drawn with other historic cases of protracted low solar activity, as judged for example by the sunspot number, such as the Dalton minimum (1790 - 1830). It has also been noted that unusual solar behavior may have started well before the 2007-2009 minimum (Feynman and Ruzmaikin, 2011). It was hypothesized that the years 2007-2009 represented a minimum of the Centennial Gleissberg Cycle, the last minimum of which occurred at the beginning of the 20th century, rather than a Maunder-type minimum (Feynman and Ruzmaikin, 2011).

Diminished solar activity will affect the properties of the solar wind and hence the types of interaction it has with planetary magnetospheres. In this paper we concentrate on the variation of the solar wind parameters and, in particular, of the energy budget in the inner heliosphere (kinetic and magnetic energy densities). Examining their effects on the terrestrial magnetosphere, we focus on two key aspects:

i) an estimate of the cross-polar cap potential in its dependence on these interplanetary parameters (i.e. the dayside source of plasma convection in the magnetosphere), and

ii) the shapes of the bow shock and magnetopause.

We also compare these findings to others reported for more typical solar activity, such as the minimum in 1995. Results on aspect i) will allow an educated guess as to the solar wind-magnetosphere interaction processes most common during this three-year period. Results on ii) - admittedly restricted to a three-month interval - will allow us to see in what way the magnetopause boundary and its shape (i.e. stand-off distance and flaring) reflect the interplanetary parameters and how they differ from three widely used models of the magnetopause.

For considerations of energies in the interplanetary medium we discuss field and plasma measurements made by STEREO-A (ST-A). For the impact on the terrestrial magnetosphere 
we use magnetic field and plasma data from the Wind spacecraft. In 2007-2009 Wind was orbiting around the Lagrangian L1 point $\sim 220 R_{\mathrm{E}}$ upstream of Earth. ST-A, launched in October 2006, is in an Earth-like orbit but leads the Earth, increasing its longitudinal separation from Earth by $22.5^{\circ}$ year. At the end of 2009 its angular separation from Earth was $\sim 68^{\circ}$. The magnetospheric response is monitored by data from Cluster 1, Geotail, and the THEMIS B and C spacecraft.

\section{Solar Wind Observations: STEREO-A}

We look at solar wind observations from the vantage point of ST-A. Figures $1-5$ give an overview of key solar wind and IMF parameters. The magnetic field and plasma data are, respectively, from the IMPACT (Luhmann et al., 2008) and PLASTIC (Galvin et al., 2008) instrument suites. The format of the figures is the same. The top panel shows the temporal profile of the relevant quantity $\left(B, V, T, N, B_{N}\right)$ at 1 -min resolution. The data set is subdivided into three samples (S1-S3) as indicated. S1: March 2007 - December 2007; S2: January 2008 - October 2008; S3: November 2008-October 2009. The subdivision into three intervals of approximately equal duration is arbitrary and is merely meant to bring out any evolutionary trends that may have occurred. The second panel shows the frequency distribution of the relevant quantity over each sample in the form of histograms. Finally, the third panel shows a spectrogram of the distribution, arranged by Carrington Rotation (CR) and month/year. The occurrence frequency is color-coded as shown on the right of this panel.

Figure 1 refers to the magnetic field strength. All three distributions are skewed to the left and exhibit long tails. The distribution is thus not Gaussian and can reasonably well be fitted by a log-normal distribution (not shown). The field peaks at $\approx 3.5 \mathrm{nT}$ and the spectrogram shows that the most probable values lie in the range $\sim 2.5-\sim 4.5 \mathrm{nT}$. The distribution is narrowest in S2 and the peak is slightly shifted to higher values there. We determine the "most probable values" for the physical parameters plotted in Figures $1-8$ from a visual inspection of the bottom panels of the respective figures.

We next consider plasma (proton) parameters. Figure 2, 3, 4 show results for the bulk speed $(V)$, temperature $(T)$, and density $(N)$, respectively. The time profiles and histograms for $V$ indicate multiple peaks in S1 and S2, also reflected in the wide spread of values in the spectrogram. In fact, the time profiles for $\mathrm{S} 1$ and $\mathrm{S} 2$, too, are indicative of a succession of alternating slow and fast streams of large amplitude with associated stream-stream interactions (co-rotating or stream interaction regions, CIRs/SIRs; Gosling and Pizzo, 1999), a pattern which stands out most clearly in S2. By contrast, the velocity profile of S3 is more spiky and the velocity range is more restricted, with few cases of bulk speeds exceeding $600 \mathrm{~km} \mathrm{~s}^{-1}$. There is a much thinner, two-peak structure in S3, centered on lower values (slow solar wind). It may be noted that these two V-peaks in S3 are very similar to the two peaks observed at low velocities in the distributions $\mathrm{S} 1$ and $\mathrm{S} 2$ - one below $300 \mathrm{~km} \mathrm{~s}^{-1}$ and the other above $300 \mathrm{~km} \mathrm{~s}^{-1}$. So, two peaks at low velocities seem to persist throughout the whole period. We thus expect to see also several stream-stream interactions where a slow stream (defined here as $V<450 \mathrm{~km} \mathrm{~s}^{-1}$ ) overtakes an even slower stream. The multiple peaks at low $V$ likely suggest different sources of the slow solar wind. In addition, S1 and S2 have a high-velocity component with extra peaks.

The proton temperature distributions (Figure 3) are skewed and possess long tails. Samples S1 and S2 indicate a broad maximum, possibly a two-peak feature. The distribution in 

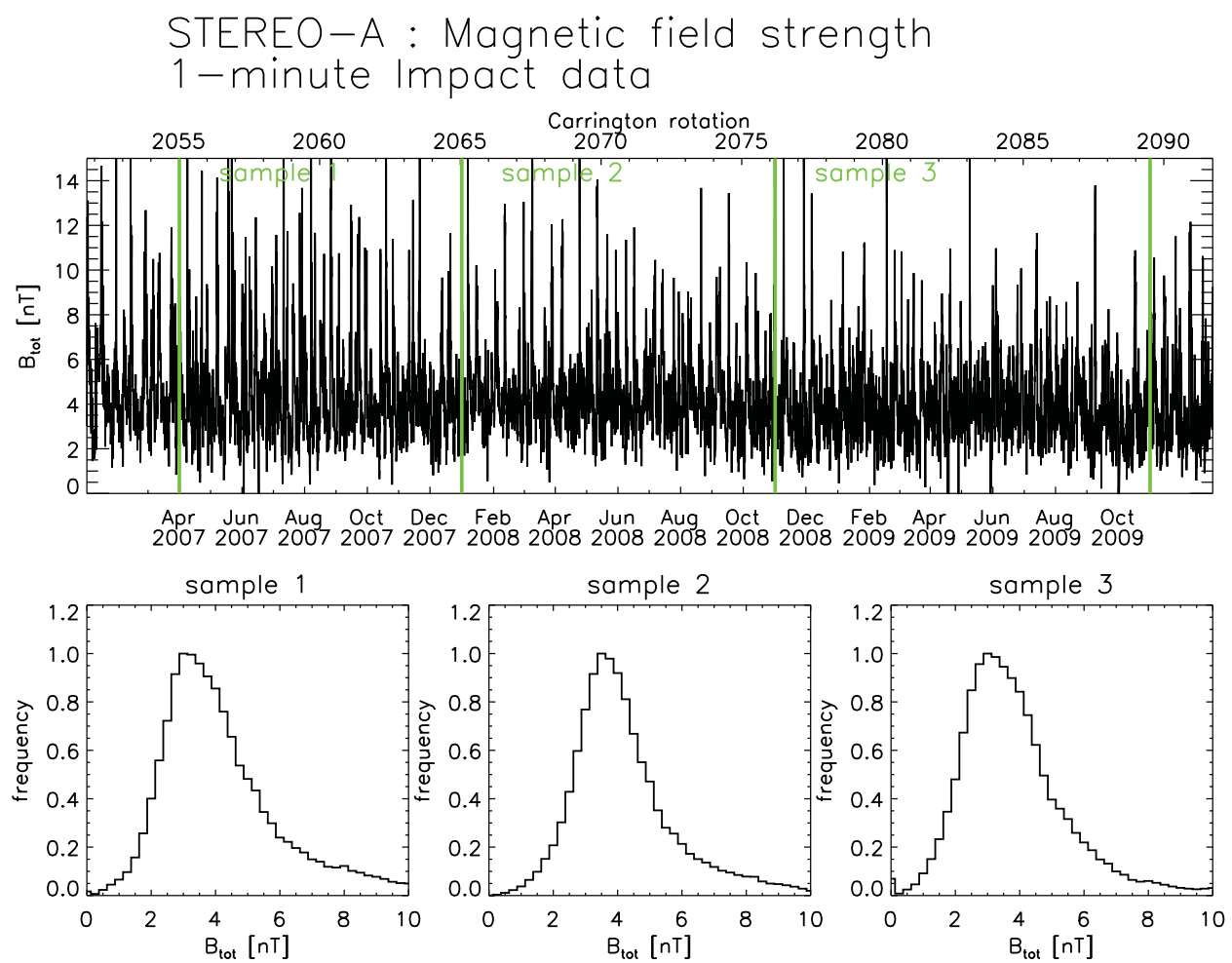

Total magnetic field strength distributions over Carrington rotation 2053-2089

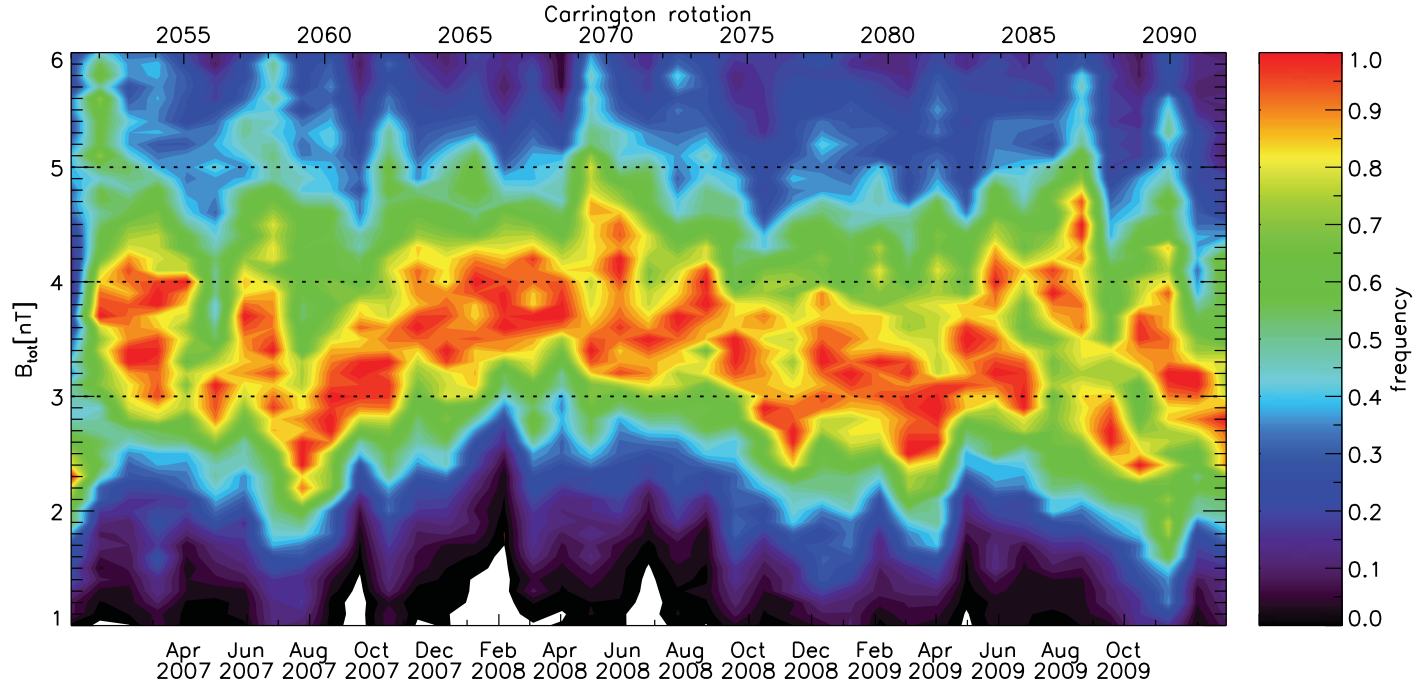

Figure 1 The distribution of the total interplanetary magnetic field at STEREO-A. The top panel shows the time series, the middle panel gives its distribution over the three times intervals (samples); and the bottom panel shows a spectrogram of its distribution as a function of Carrington rotation and month/year.

$\mathrm{S} 3$ is shifted to lower values. Most probable values lie in the ranges $2.5-12 \times 10^{4} \mathrm{~K}$ (S1), $3-16 \times 10^{4} \mathrm{~K}(\mathrm{~S} 2)$, and $2-9 \times 10^{4} \mathrm{~K}(\mathrm{~S} 3)$.

Figure 4 shows results for the proton density. The distributions are strongly skewed to the left and maxima are reached at very low values, particularly on S1 and S2. The narrowest distribution occurs in S2. The highest densities are reached in S3 because, as we see from the distributions of each sample, the most probable value is shifted to higher values from S1 


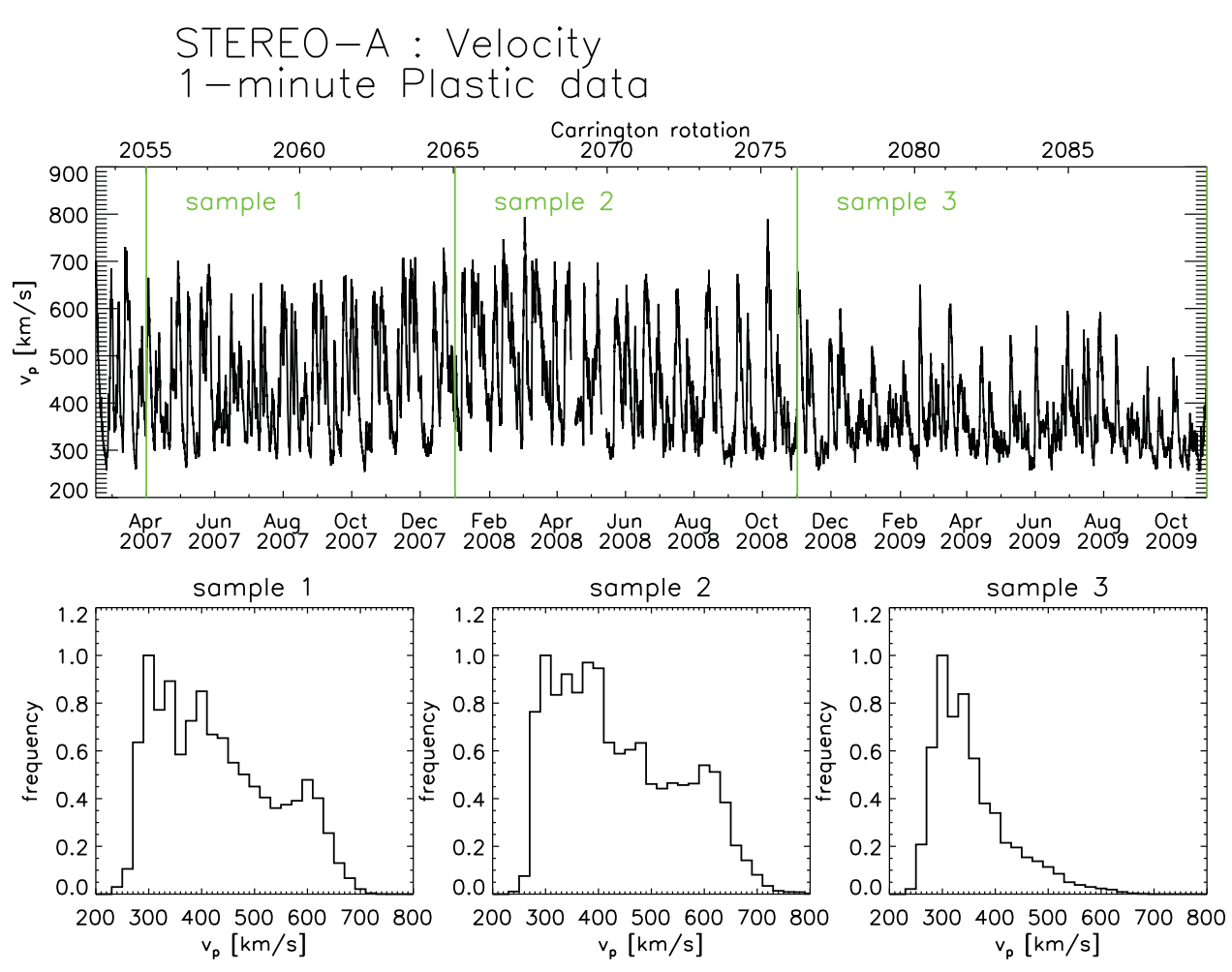

Velocity distributions over Carrington rotation 2053-2089

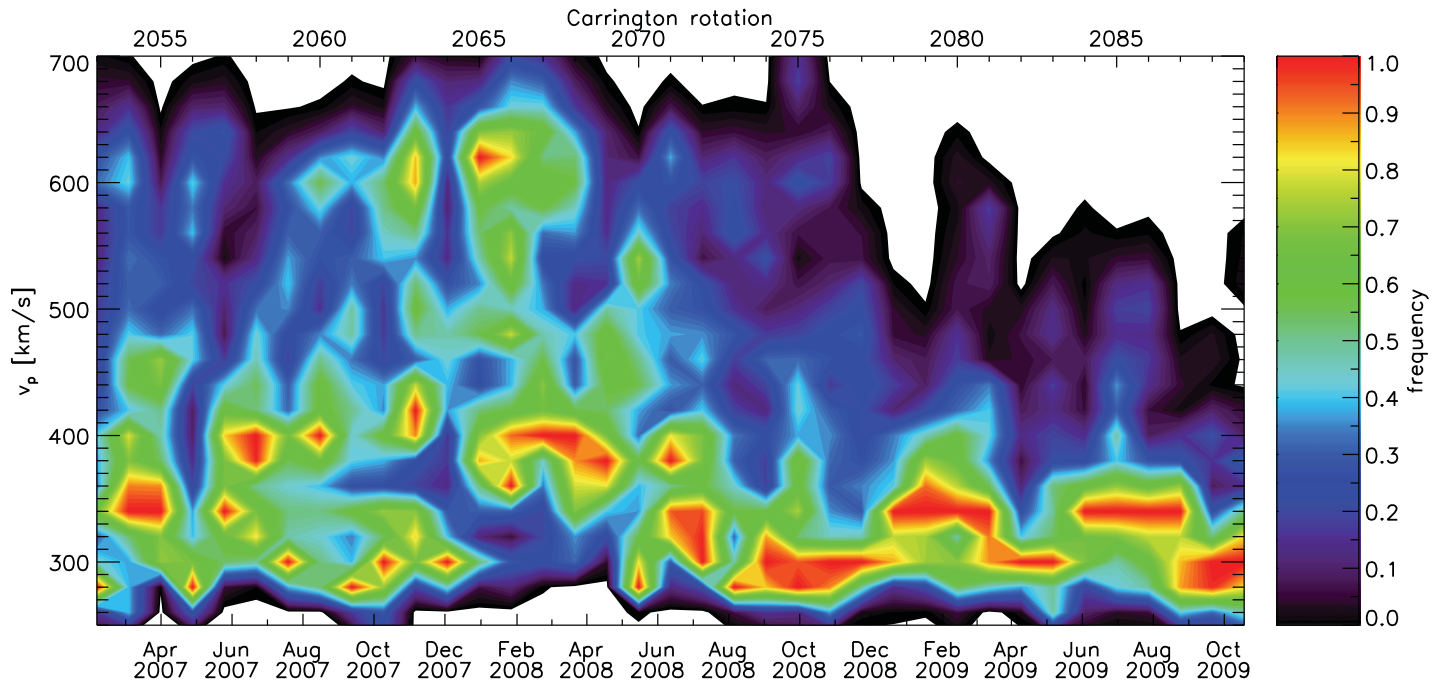

Figure 2 Similar to Figure 1, but for the solar wind bulk speed.

to S3 (bottom panel), although the differences between S1 and S3 are relatively small. Most probable values lie in the ranges $1-5 \mathrm{~cm}^{-3}(\mathrm{~S} 1) ; 1-3 \mathrm{~cm}^{-3}$ (S2), and $2-6 \mathrm{~cm}^{-3}$ (S3).

Figure 5 shows the distribution of the $B_{N}$ component of the magnetic field. $R T N$ coordinates are used. In these coordinates unit vector $\mathbf{R}$ points from the Sun to the spacecraft (here, ST-A), vector $\mathbf{T}$ points in the direction of planetary motion, and unit vector $\mathbf{N}$ completes the right-handed $R T N$ system and is such that the $R N$ plane contains the rotation axis of the Sun. The distributions in all three samples are similar and may be modeled by Gaussian (normal) distributions centered around 0. This is quite typical behavior. Not so typical are the narrow profiles (compare with Figure 13 in Luhmann et al. (1993) for solar minimum 


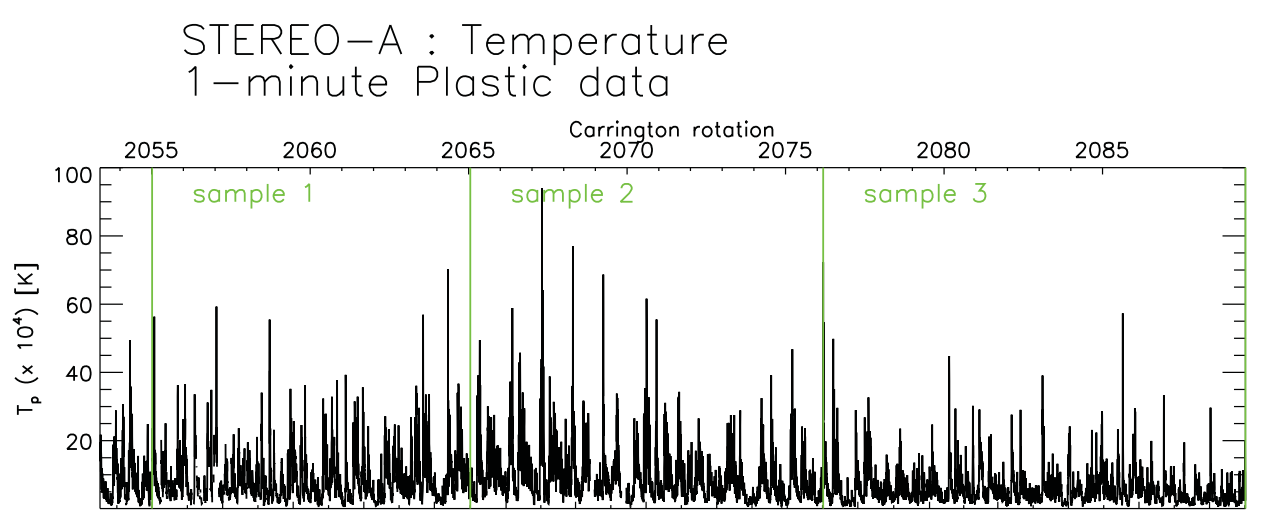

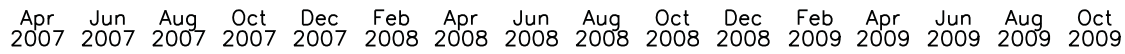
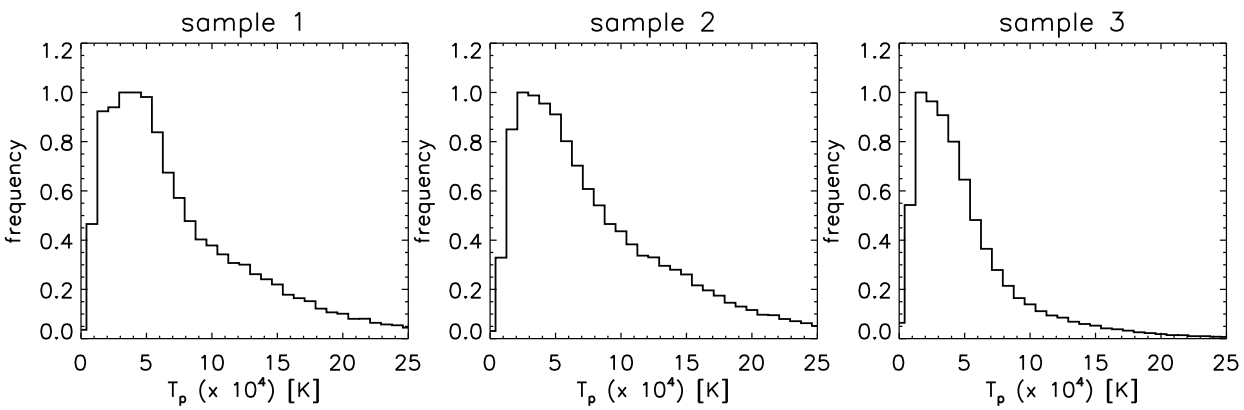

Velocity distributions over Carrington rotation 2053-2089

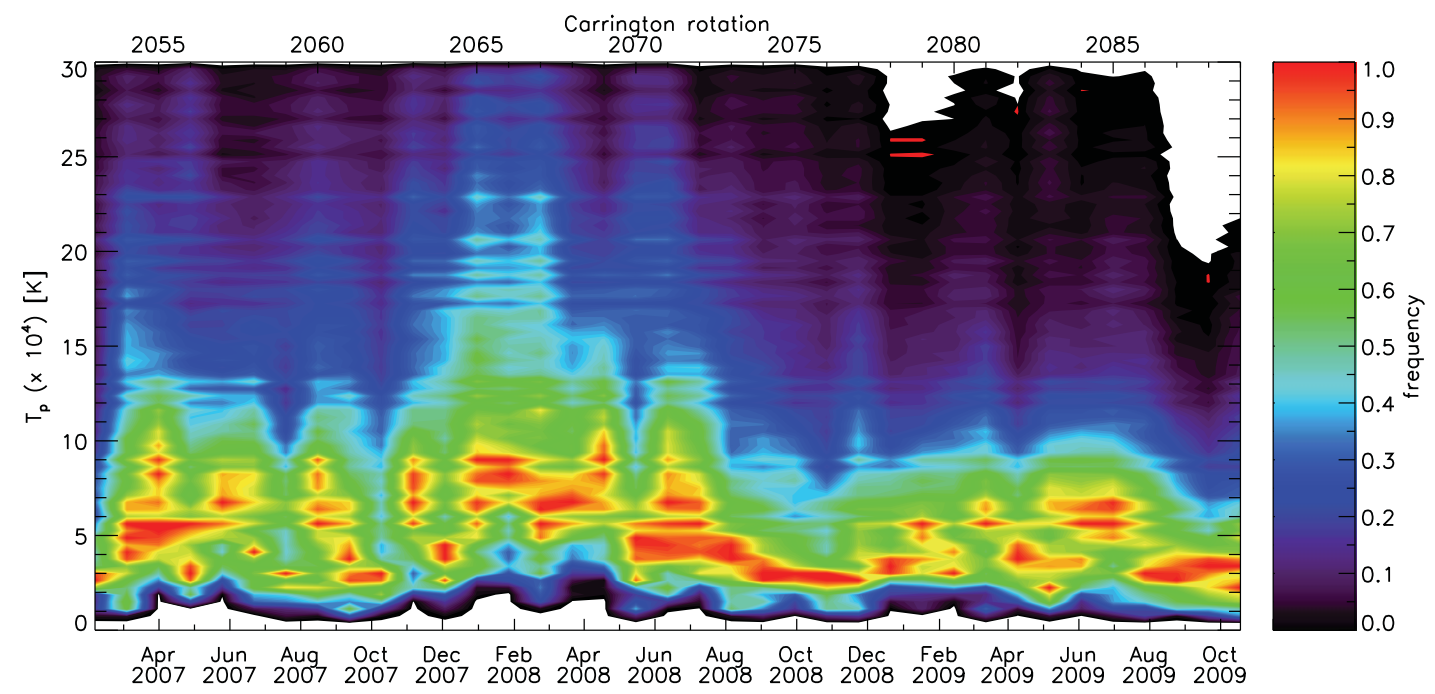

Figure 3 Similar to Figure 1, but for the solar wind proton temperature.

conditions in 1975), as may also be seen from the thin slice around $0 \mathrm{nT}$ where the most frequent values are clustered and which is of width $\sim 3 \mathrm{nT}(\sim-1.5$ to $1.5 \mathrm{nT})$. This behavior is similar for GSM $B_{z}$ at Wind (not shown). Since $B_{N}\left(B_{z}\right)$ is the major component in determining the magnetic shear at the magnetopause, and hence the likelihood of reconnection there, the ST-A and Wind data suggest that reconnection processes, while certainly present, were not a dominant aspect of the interaction of this solar wind with the magnetosphere.

We support the last statement further by a study of solar wind-magnetosphere coupling functions. To be specific, we consider the Kan-Lee reconnection electric field ( $E_{\mathrm{KL}}$, Kan and Lee, 1979; see also Sonnerup, 1974; Hairston, Hill, and Heelis, 2003), which includes $B_{z}$, 

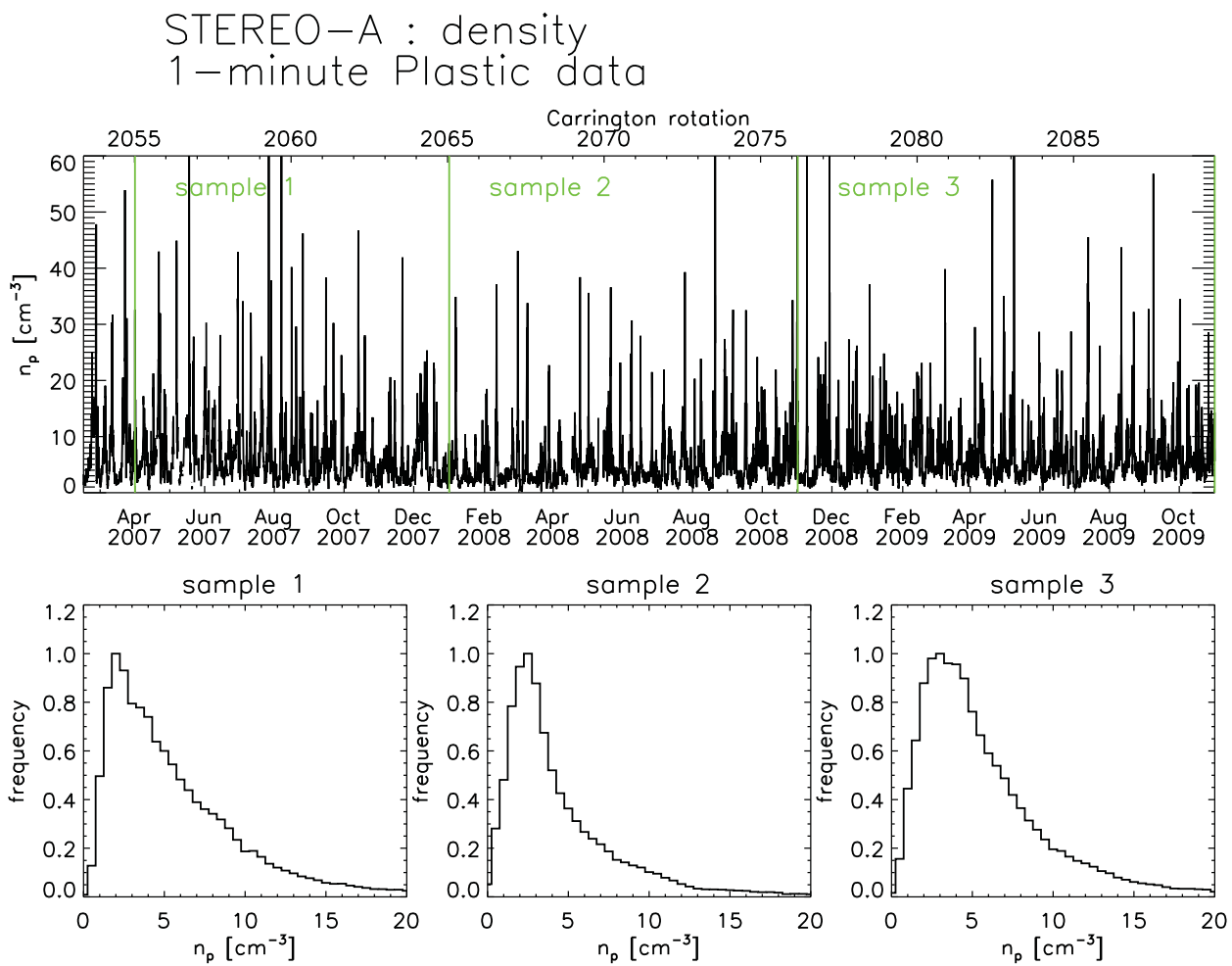

Density distributions over Carrington rotation 2053-2089

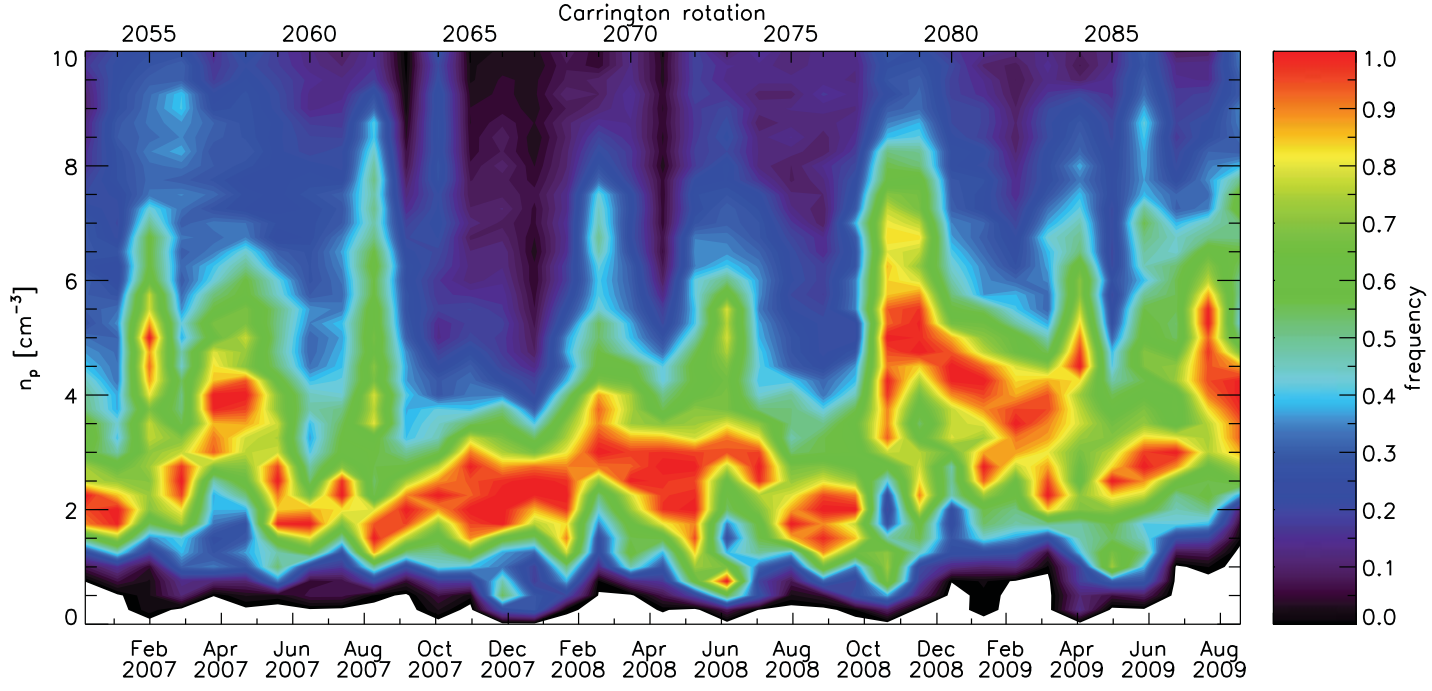

Figure 4 The distribution of the solar wind proton density from ST-A.

$B_{y}$ and the IMF clock angle, $\theta$ (i.e., the polar angle in the GSM $Y Z$ plane). It is defined as $E_{\mathrm{KL}}=V B_{\tau} \sin ^{2}(\theta / 2)$, where $B_{\tau}=\left(B_{y}^{2}+B_{z}^{2}\right)^{1 / 2}$. We use OMNI data (King and Papitashvili, 2005). Quantity $E_{\mathrm{KL}}$ follows a log-normal distribution. Over the three-year period $2007-$ 2009 we find that the most probable value $E_{\mathrm{KL}}=0.28 \mathrm{mV} / \mathrm{m}$ with a multiplicative standard deviation of 0.64 . For comparison we did the same analysis for three years of solar activity maximum 2001-2003 and obtained $0.61 \mathrm{mV} / \mathrm{m}$ with a multiplicative standard deviation of 0.66 . While the width of the $E_{\mathrm{KL}}$ distributions is thus quite similar (multiplicative factor 0.66 and 0.64 ), the most probable value in $2007-2009$ is about one-half its value in $2001-$ 2003. 


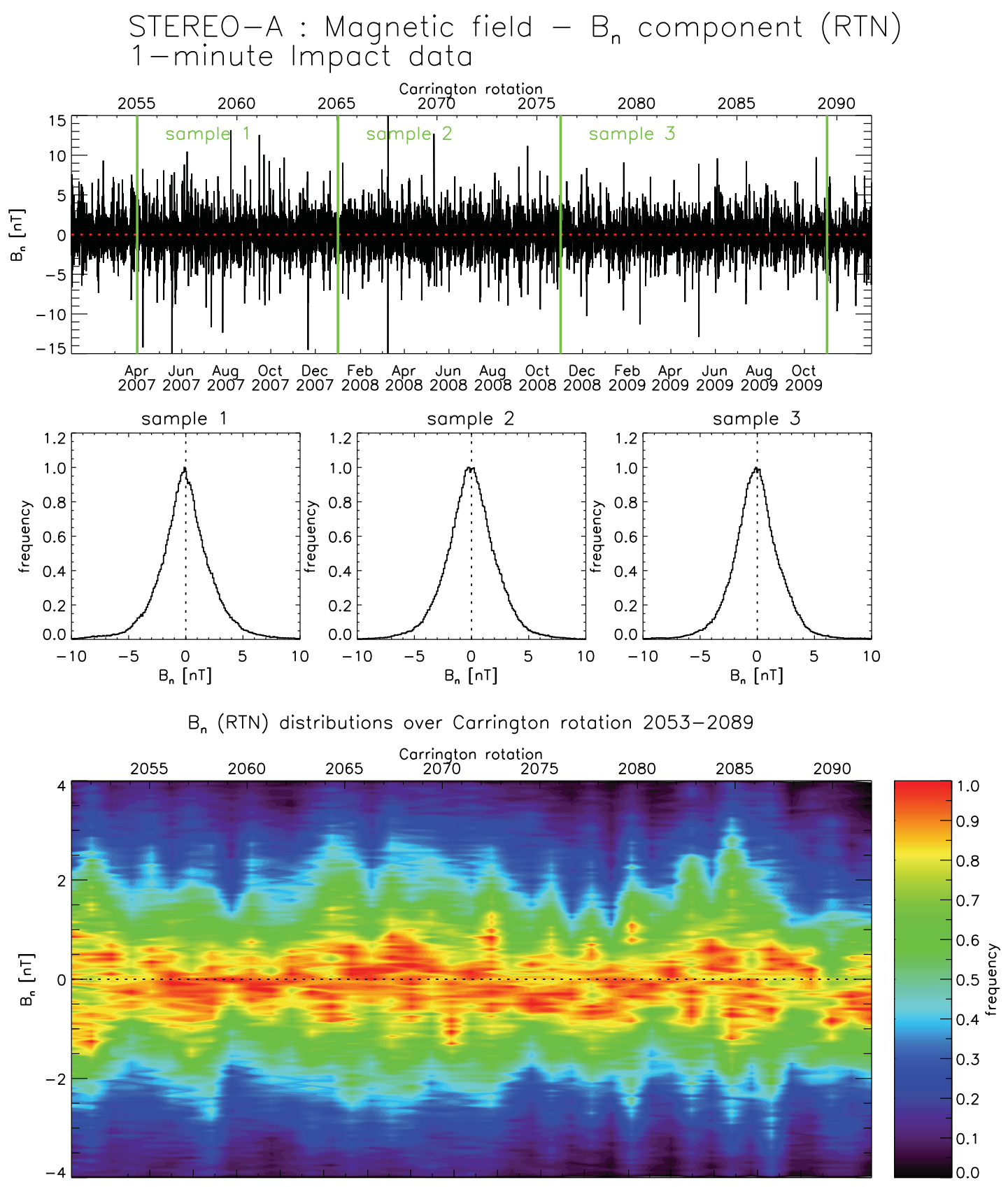

Apr Jun Aug Oct Dec Feb Apr Jun Aug Oct Dec Feb Apr Jun Aug Oct
2007200720072007200720082008200820082008200820092009200920092009

Figure 5 The distribution of the $B_{N}$ component of the magnetic field. $R T N$ coordinates are used.

\subsection{Comparison with Solar Minimum $1995-1996$}

We now compare some aspects of the distributions in 2007-2009 with those in the previous minimum (Cycle 22/23), from mid 1995 - mid 1996. We start with the three plasma parameters for which we compare with results given by Burlaga and Lazarus (2000). In 1995 many co-rotating interaction regions were present (see for example their Figure 2), very similar to the velocity profile in 2007-2008. All proton plasma parameters $V, N$ and $T$ in 1995 had double-peaked distributions. On this we have agreement in the $V$ and, though less pronounced, in the $T$ distributions, but this feature is not so evident in the $N$-distributions for 


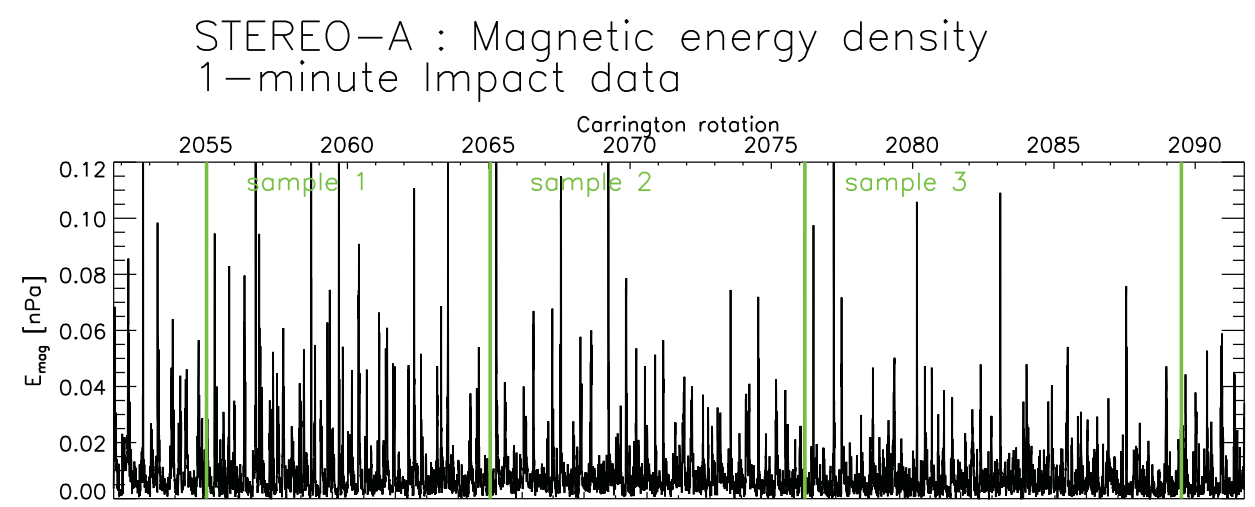

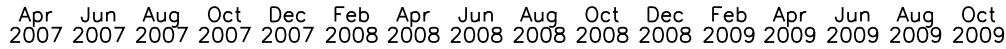
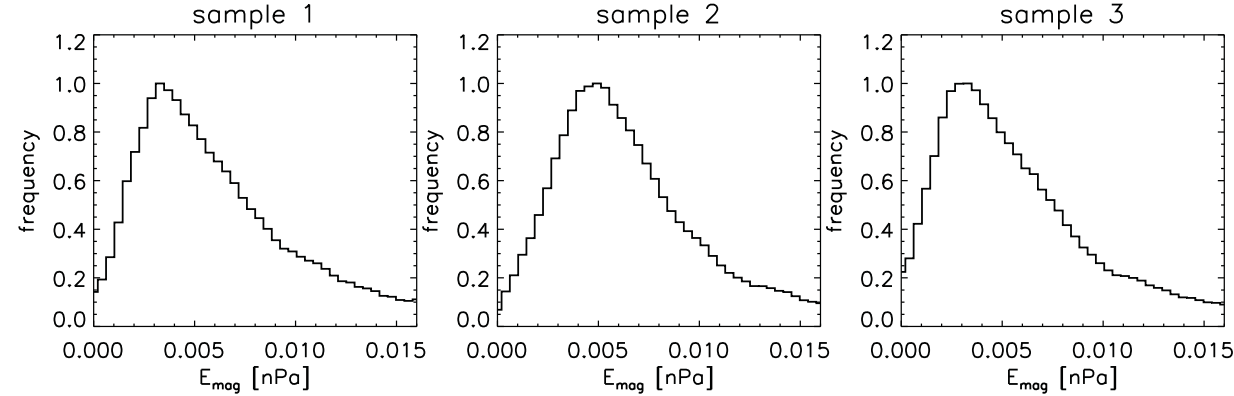

Magnetic energy density distributions over Carrington rotation 2053-2089

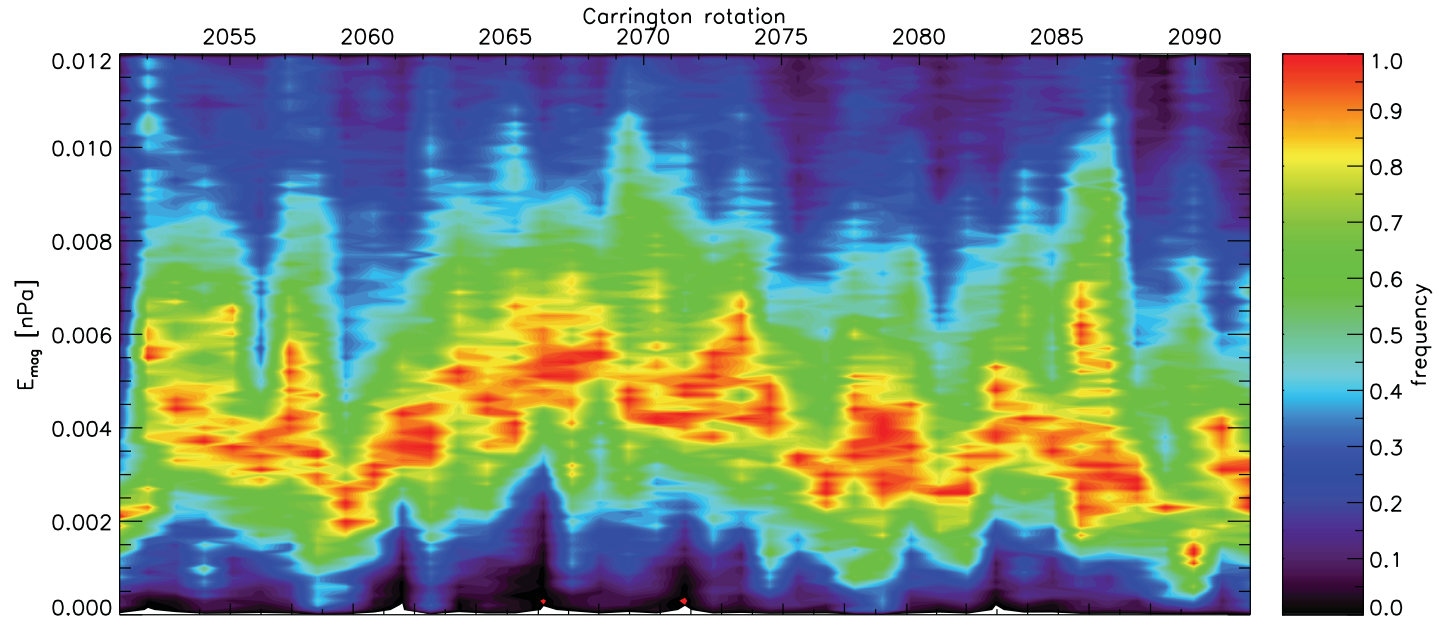

Apr Jun Aug Oct Dec Feb Apr Jun Aug Oct Dec Feb Apr Jun Aug Oct
2007200720072007200720082008200820082008200820092009200920092009

Figure 6 The distribution of solar wind magnetic energy densities.

reasons not clear to us. We shall thus compare their most probable values in the two peaks for $V$ and in only the major peak for $T$ and $N$. For 1995, Burlaga and Lazarus (2000) find most probable values of

i) $377 \mathrm{~km} \mathrm{~s}^{-1}$ and $618 \mathrm{~km} \mathrm{~s}^{-1}$ for $V$;

ii) $5.5 \times 10^{4} \mathrm{~K}$ for $\mathrm{T}$; and

iii) $8.3 \mathrm{~cm}^{-3}$ for $N$.

Comparing with our results, we have similar values for $V$ and $T$, particularly in S1 and S2. The large divergence occurs in $N$ where we record much lower densities $(\sim 3-4$ 


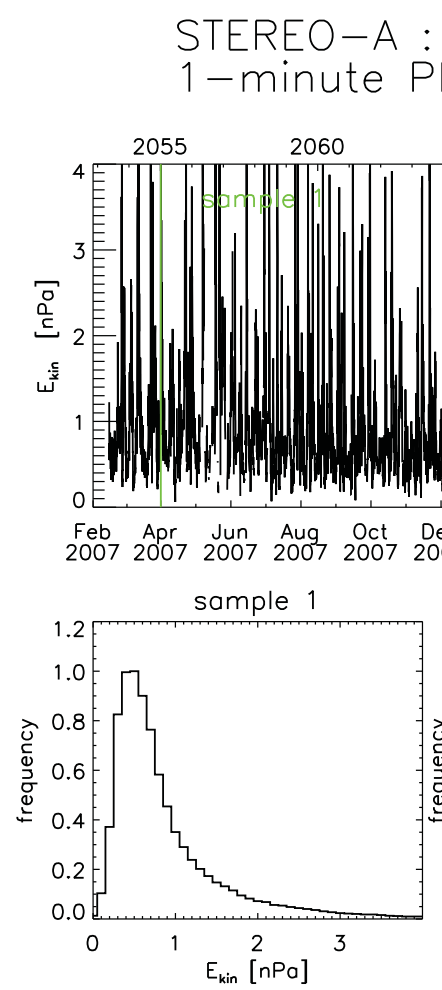

STEREO-A : kinetic energy density

1 -minute Plastic data

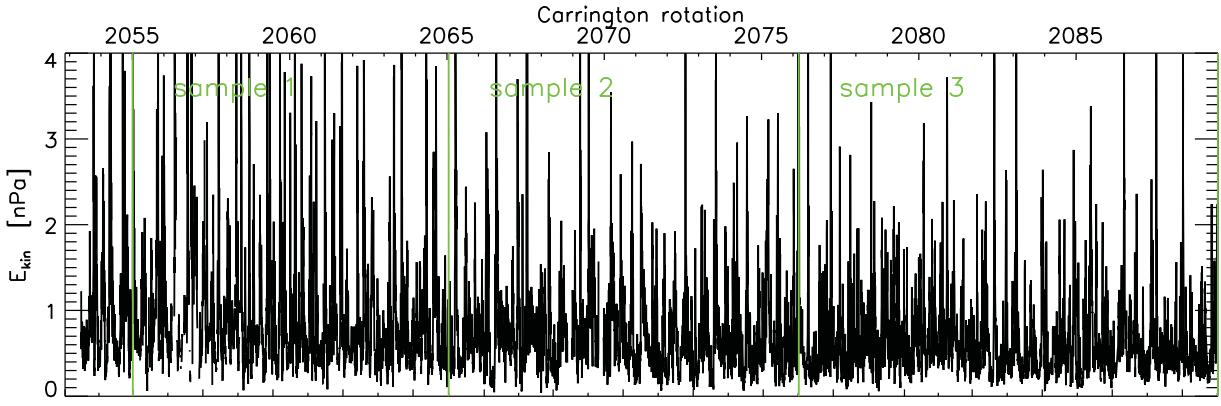

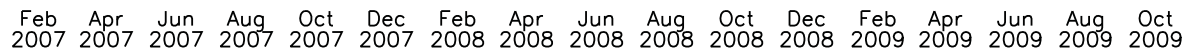
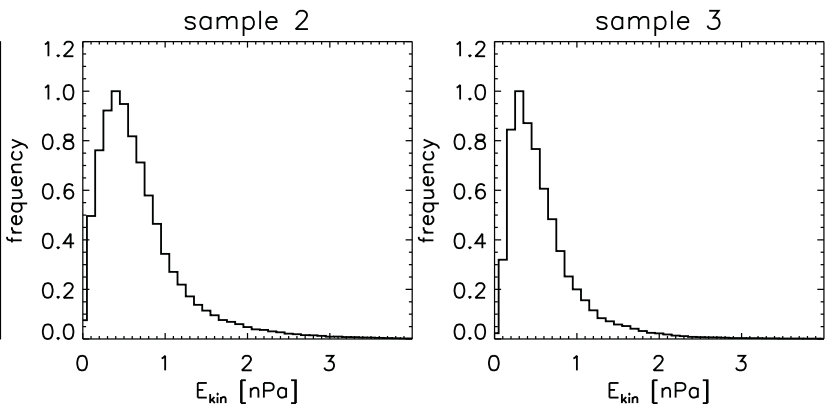

Kinetic energy distributions over Carrington rotation 2053-2089

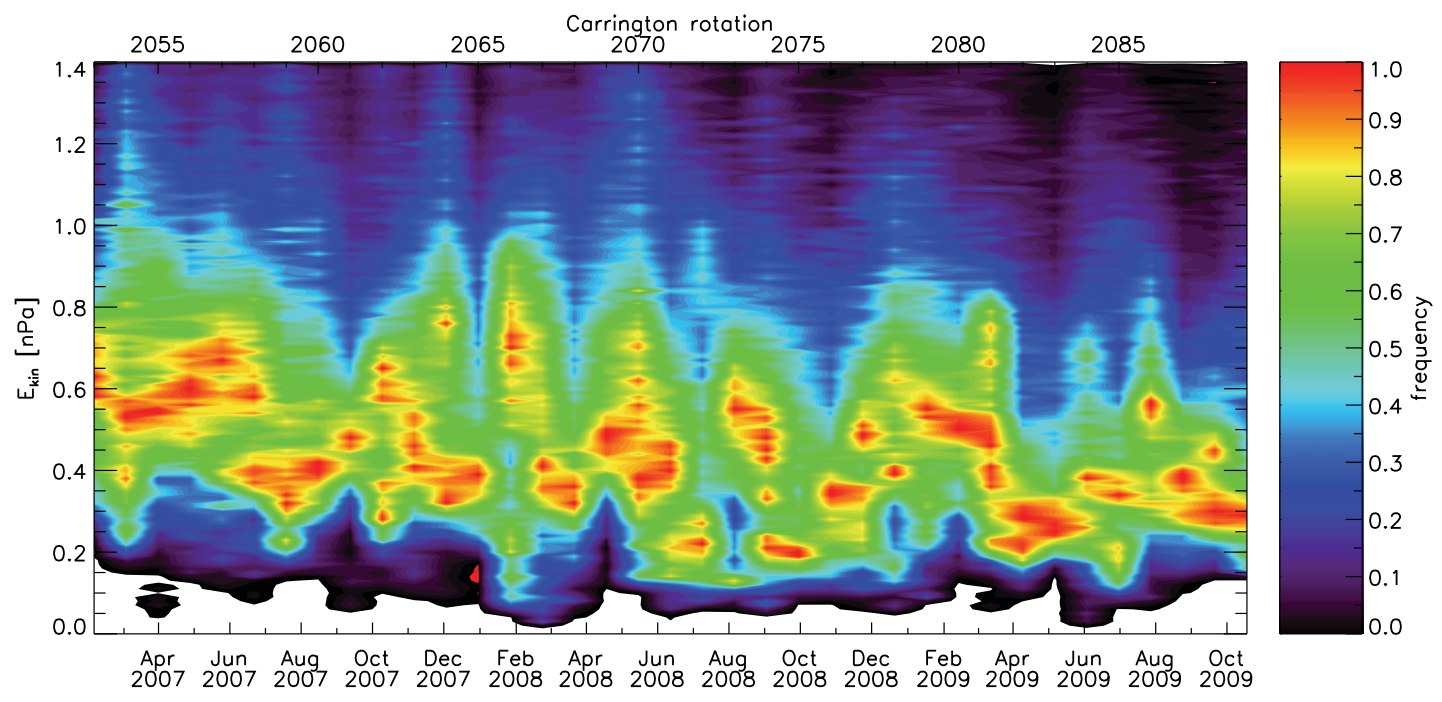

Figure 7 Similar to Figure 6 but for the solar wind kinetic energy densities.

vs. $8 \mathrm{~cm}^{-3}$ ). The density is thus the interplanetary plasma parameter which deviates most from the previous minimum.

We now consider the magnetic field in the previous solar minimum. For this we use 1-min averages from the OMNI database for May 1995-May 1996. We obtain (average and standard deviation) $B=5.4 \pm 2.5 \mathrm{nT}$ and for $B_{z}:-0.4 \pm 3.0 \mathrm{nT}$. For comparison, the most probable values of $B$ during the 2007-2009 solar minimum lie in the range [2.54.5] $\mathrm{nT}$ and the those of $B_{z}$ in the range [ $-1.5,1.5 \mathrm{nT}$ ]. So the magnetic field strength is significantly lower in 2007-2009. Low magnetic field strengths favor the growth of the Kelvin-Helmholtz instability at the magnetopause (see Section 5). 
STEREO-A : quasi invariant

1-minute combined Plastic and Impact data

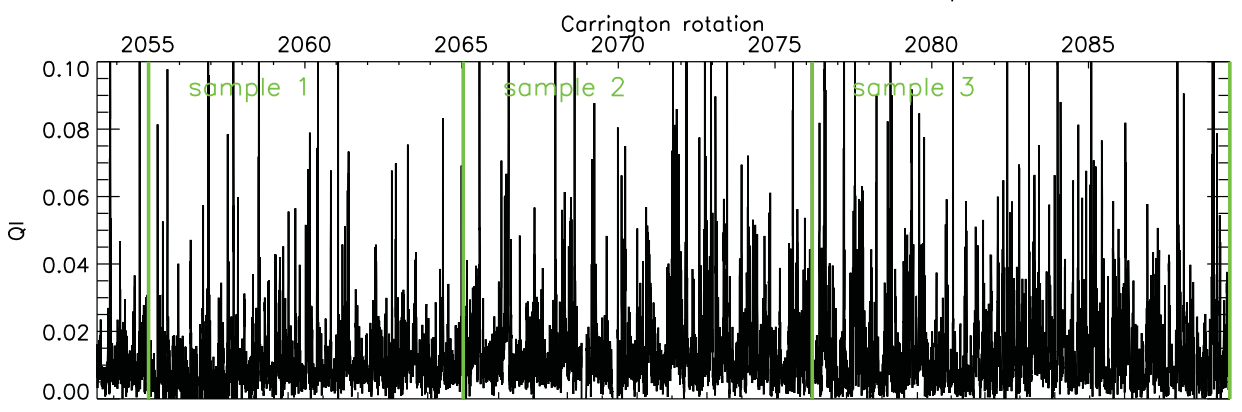

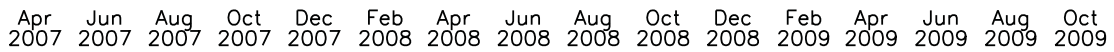
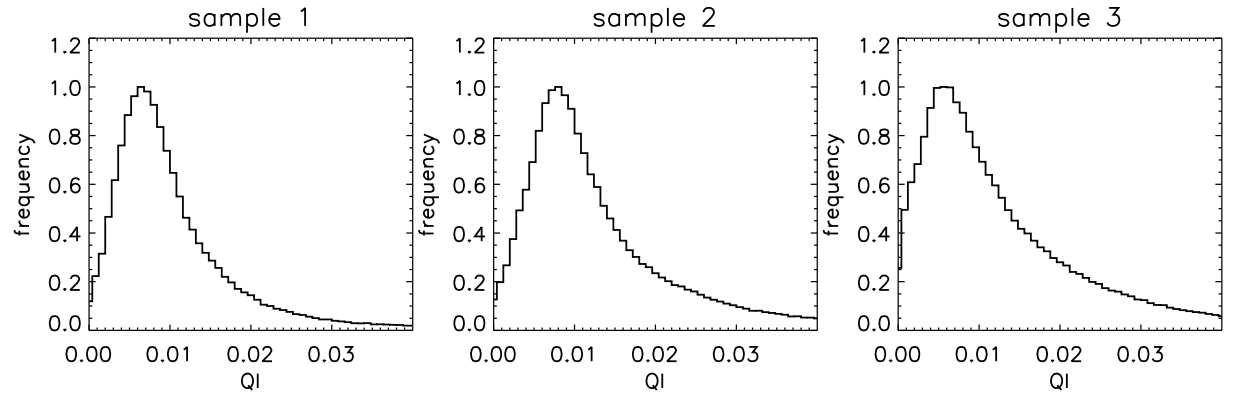

Q| distributions over Carrington rotation 2051-2089

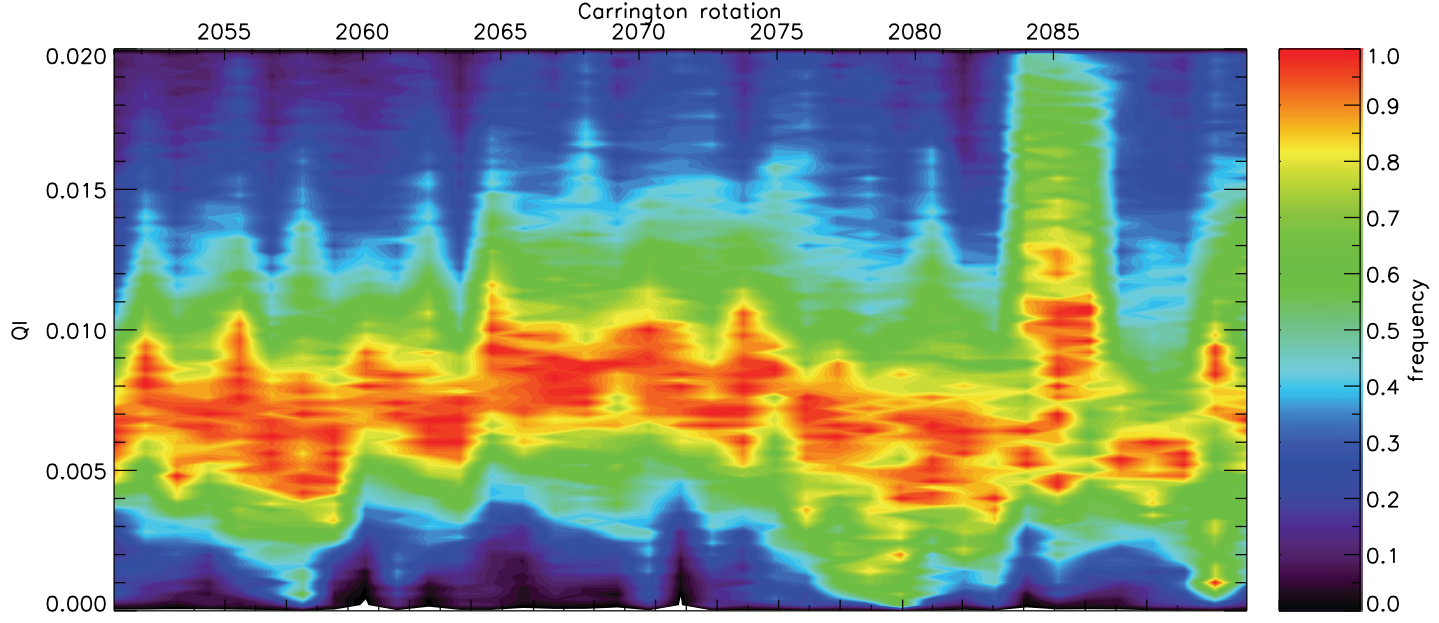

Apr Jun Aug Oct Dec Feb Apr Jun Aug Oct Dec Feb Apr Jun Aug Oct
2007200720072007200720082008200820082008200820092009200920092009

Figure 8 Distribution of the ratio of the magnetic-to-kinetic energy densities, i.e., the solar wind quasi-invariant, QI. Note the significant rise during CRs 2285-2287.

\subsection{Solar Wind Energy Densities}

We next discuss the solar wind energy densities and their ratio, the so-called "solar wind quasi-invariant", which was introduced by Osherovich, Fainberg, and Stone (1999). Figures 6 and 7 show the magnetic $\left(E_{\mathrm{mag}}=B^{2} / 2 \mu_{0}\right)$ and kinetic $\left(E_{\mathrm{kin}}=1 / 2 m_{p} N V^{2}\right)$ energy densities, respectively, where $m_{p}$ is the proton mass. The distributions of $E_{\text {mag }}$ feature long tails and are of the log-normal variety (Leitner et al., 2009). The most probable values lie in the ranges $\sim 2-6 \times 10^{-3} \mathrm{nPa}(\mathrm{S} 1) ; 3-7 \times 10^{-3} \mathrm{nPa}(\mathrm{S} 2)$, and $2-7 \times 10^{-3} \mathrm{nPa}(\mathrm{S} 3)$. 
The kinetic energy density distributions in Figure 7 also have long tails and are skewed to the left similar to other plasma parameters. The first two data samples feature broader distributions, reflecting a similar feature of the velocity distribution (Figure 2). The most probable values lie in the ranges $0.3-0.7 \mathrm{nPa}(\mathrm{S} 1), 0.2-0.8 \mathrm{nPa}(\mathrm{S} 2), 0.2-0.6 \mathrm{nPa}(\mathrm{S} 3)$.

The ratio $E_{\mathrm{mag}} / E_{\text {kin }}$ - the so-called quasi-invariant, QI - is plotted in Figure 8. The relevance of quantity QI to our considerations is that it has been shown to correlate very well with solar activity as measured by the sunspot number (SSN), better than the individual parameters making up this ratio. It is thus a good proxy for solar activity which, furthermore, has the distinct advantage of being based on local in situ interplanetary measurements. The correlation with SSN has been found to be very good at 1 AU (Osherovich, Fainberg, and Stone, 1999), at large AUs in the outer heliosphere (Fainberg and Osherovich, 2002) and, using Pioneer Venus (Fainberg, Osherovich, and Stone, 2001) and Helios 1 and 2 data (Leitner et al., 2005) also within $1 \mathrm{AU}$. QI is related to the Alfvén Mach number, $M_{\mathrm{A}}$, by $\mathrm{QI}=M_{\mathrm{A}}^{-2}$. QI distributions for the normal solar wind have been modeled well by log-normal distributions (Leitner, Farrugia, and Vörös (2011) and references therein). Most frequent values lie in the range [0.004, 0.010], corresponding to the $M_{\mathrm{A}}$ range $[15.8,10.0]$, which is typically higher than $M_{\mathrm{A}}$ 's in the normal solar wind at $1 \mathrm{AU}$ (see e.g. Slavin and Holzer, 1981). Departures from this trend may be seen in the decrease during CRs 2075-2084 and the sharp rise in 2085-2087, which could reflect some ongoing processes on the Sun (see further in Section 3).

\section{Solar Observations}

Figure 9 shows the daily international relative sunspot number (2007-2009) and its frequency distribution. The data are taken from the NASA/Goddard Space Flight Center website and are computed as a weighted average of measurements made from a network of more than 25 observatories (Clette et al., 2007). The deep minimum of Solar Cycle 23 is reflected in the low sunspot number. Over the whole period there were very few sunspots, or no sunspots at all.

Figure 10 shows the evolution of the full-disk net magnetic flux density (in $\mu \mathrm{T}$ ) of the Sun, taken from the Stanford Solar Observatory (see Scherrer et al., 1977), together with its frequency distribution. The full-disk integrated data reflect the combined contributions from the quiet Sun, active regions and coronal holes, and are a measure of the total "open" magnetic flux on the Sun. The open magnetic flux originates in active regions but in the course of a solar cycle it is redistributed over the solar surface by transport processes, eventually forming the polar coronal holes (Wang, 2009). Coronal holes are low-density regions of the solar corona which contain open magnetic fields along which plasma escapes into the interplanetary space forming the high-speed solar wind. The total open magnetic flux varies roughly as the Sun's total dipole strength, which tends to peak a few years after sunspot maximum (Wang, 2009). The quasi-periodic short-term fluctuations of the net magnetic flux density in Figure 10 are due to the rigid solar rotation of coronal holes with a mean synodic period of about 27 days. Due to the slow evolution and long lifetimes (over several solar rotations) of coronal holes, the open flux contribution of individual large coronal holes is well reflected in the full-disk integrated data.

Wang (2009), measuring the magnetic flux at $1 \mathrm{AU}$ for which it is reduced by a factor of $10^{4}$, notes that the open flux density in the period 2008-2009 (with three-month averages of $\sim 1.5 \mathrm{nT}$ at $1 \mathrm{AU})$ is significantly smaller than in previous sunspot minima $(\sim 2 \mathrm{nT})$. The much smaller fluctuations in S3 (2009) with an almost Gaussian distribution as compared 
sunspot number - daily averages
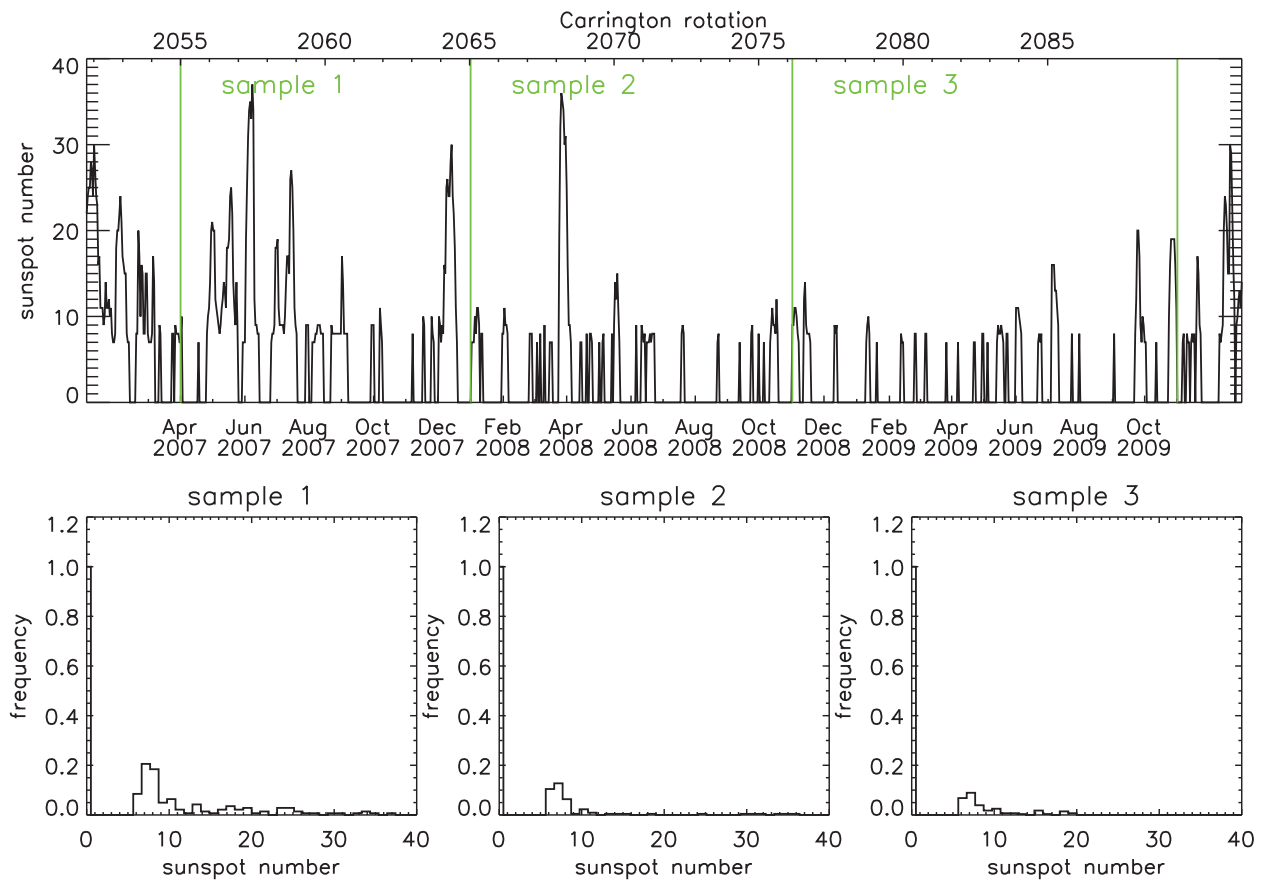

sunspot number over Carrington rotation 2051-2091

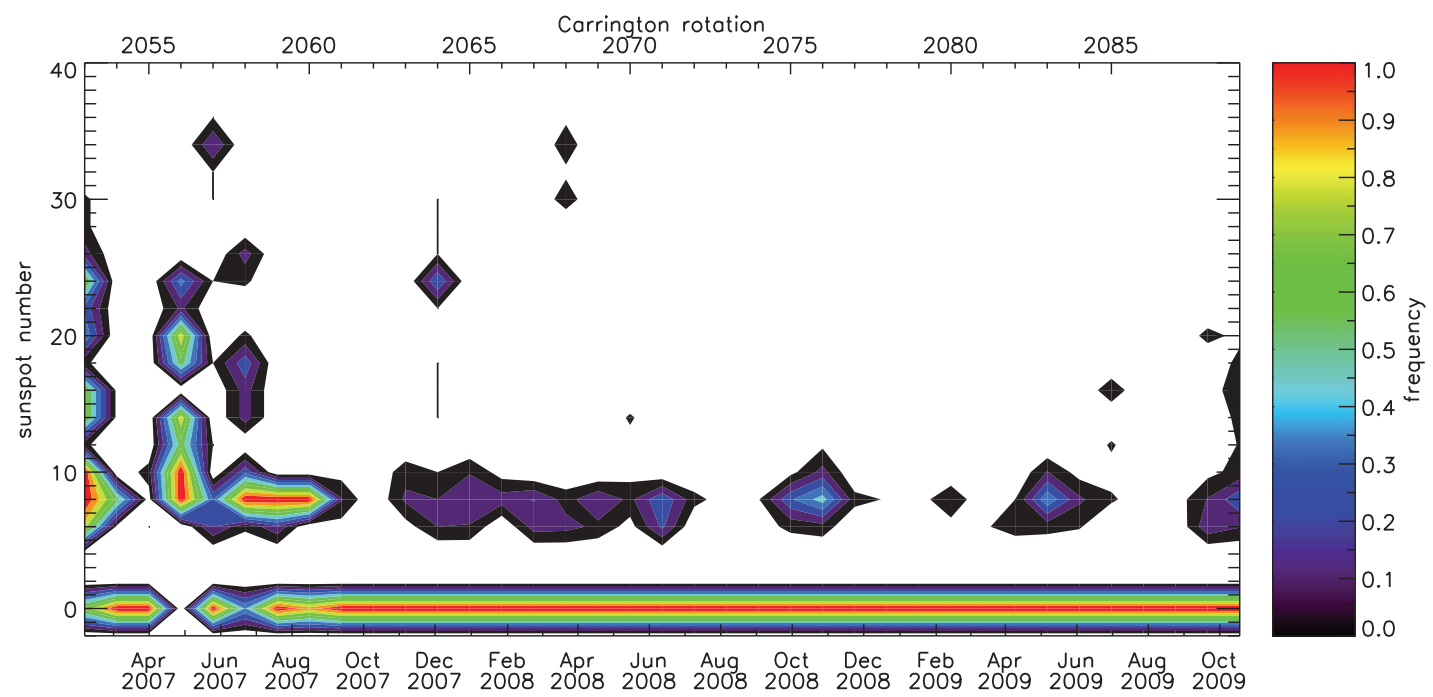

Figure 9 Evolution and distribution of the daily international sunspot number for the years 2007-2009.

to S1 and S2 (2007-2008) suggest that this trend becomes even more pronounced in the year 2009.

It may be noted that Figure 8 shows a distinct increase in the energy ratio during CR 2085-2087. This might reflect processes on the Sun. In this regard we note that during CR 2076-2079 a coronal hole (CH) which could be attributed to the new Solar Cycle 24 emerged and subsequently merged with the near northern polar $\mathrm{CH}$. The new-cycle $\mathrm{CH}$ at high latitudes contributes to a quite slow solar wind speed (maximum speeds of about $400 \mathrm{~km} \mathrm{~s}^{-1}$ ). During CR 2085-2087 two new-cycle CHs emerged and merged with the polar $\mathrm{CH}$ to the north. In addition, a $\mathrm{CH}$ with opposite polarity appeared close to this entity. 
Sun : magnetic flux (daily averages)
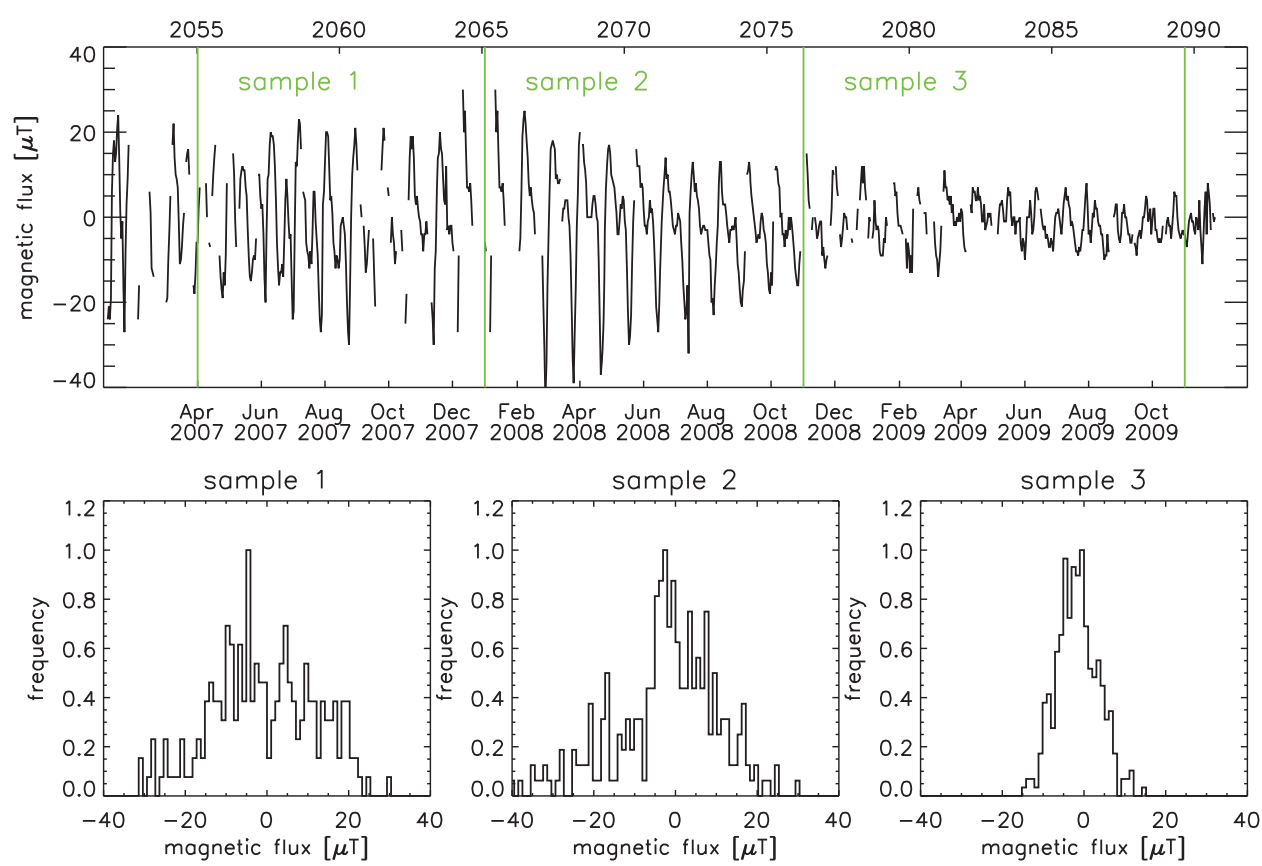

magnetic flux over Carrington rotation 2051-2091

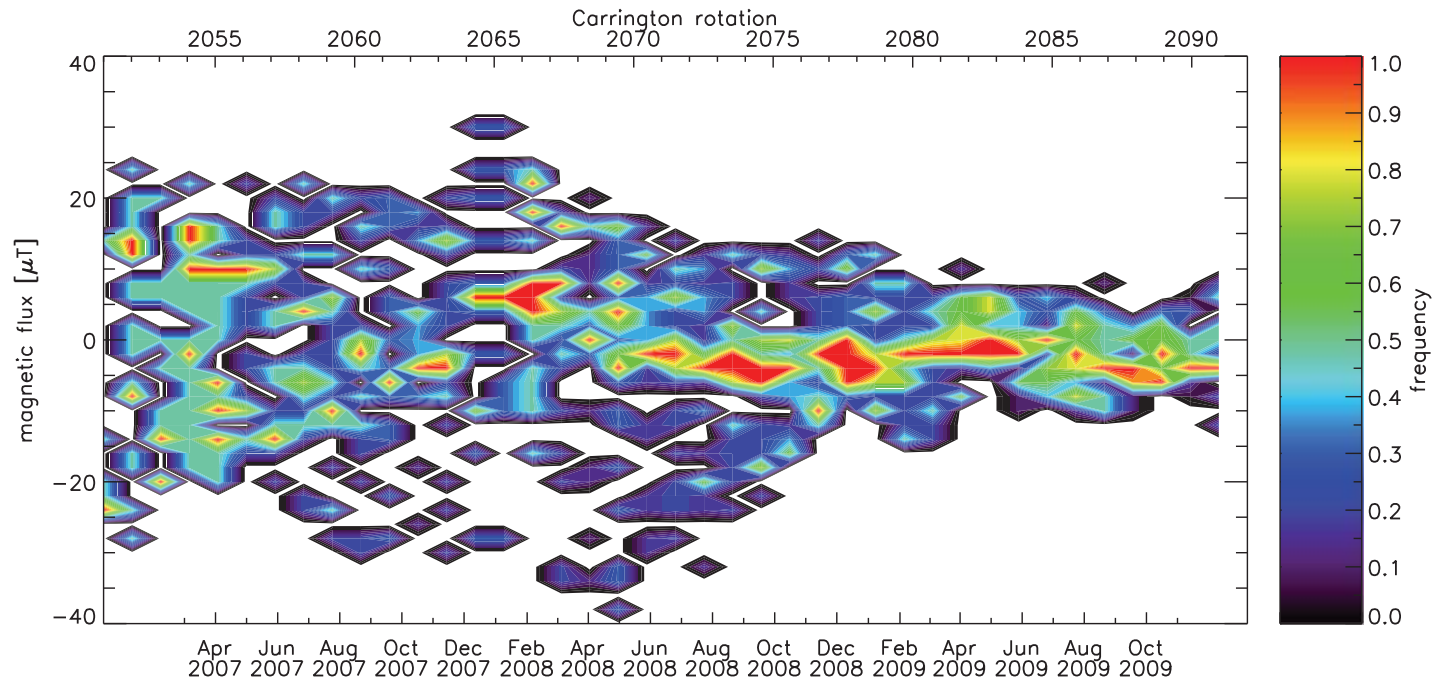

Figure 10 Similar to Figure 9, but for daily averages of the full-disk net magnetic flux density.

The complex system of northern polar $\mathrm{CH}$ extension resulted in solar wind streams with maximum speeds of about $500 \mathrm{~km} \mathrm{~s}^{-1}$ (Wang et al., 2010).

\section{Major Effects on the Magnetosphere}

\subsection{The Average Cross-Polar Cap Potential in 2007-2009}

We now discuss two major effects this solar activity minimum had on the magnetosphere. We consider first the solar wind electric field, defined by $E_{\mathrm{SW}}=\left|\mathbf{V}_{\mathrm{SW}} \times \mathbf{B}_{\mathrm{SW}}\right|$ and shown in 


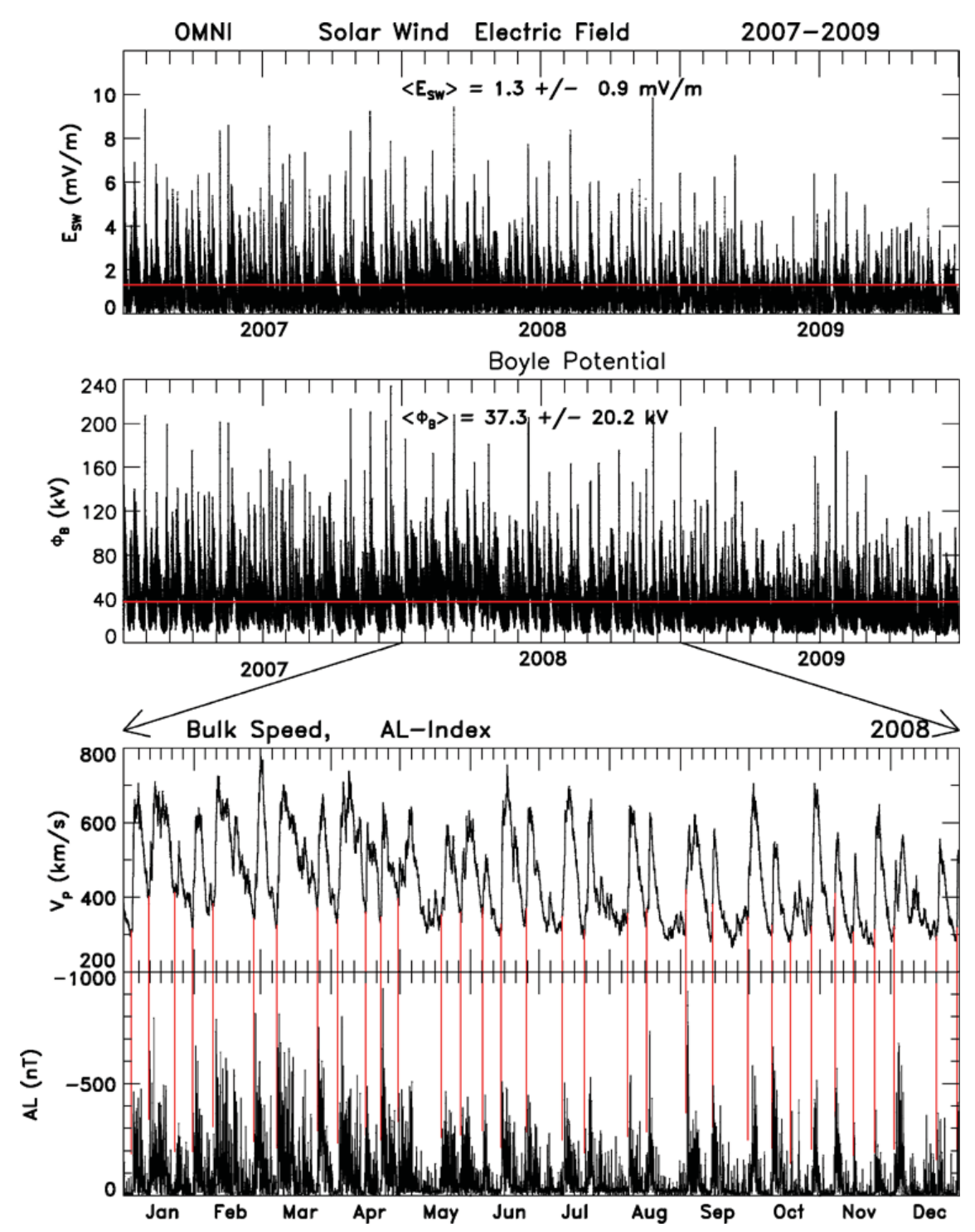

Figure 11 Over the three-year period, the top panel shows the dawn-dusk motional electric field in the solar wind $\left(V_{x}\left(B_{z}^{2}+B_{y}^{2}\right)^{1 / 2}\right)$, the second panel shows the cross-polar cap potential (CPCP) after Boyle, Reiff, and Hairston (1997); the third panel shows the bulk speed profile in 2008; and the fourth panel auroral, the electrojet AL index. Red guidelines connect stream interaction regions with enhancements in AL activity.

the top panel of Figure 11. We assume that the velocity of the solar wind is in the $-x$ direction. One-min averages are used. Average and standard deviation are $E=1.3 \pm 0.9 \mathrm{mV} / \mathrm{m}$. This is much below typical values in interplanetary coronal mass ejections (ICMEs), which are in the range of several $\mathrm{mV} \mathrm{m}^{-1}$ to a few tens $\mathrm{mV} \mathrm{m}^{-1}$ (e.g. Hairston, Hill, and Heelis, 2003; Hairston, Drake, and Skoug, 2005). The values of $E_{\text {SW }}$ shown in the figure are also generally below values when the magnetospheric response to interplanetary driving becomes non-linear, which are estimated to be in the range 6-10 $\mathrm{mV} \mathrm{m}^{-1}$ (Siscoe et al., 2002; Hairston, Hill, and Heelis, 2003; Mühlbachler et al., 2005). Thus saturation levels in magnetospheric response (for example, of the cross-polar cap potential, $\mathrm{CPCP}$ ) are not reached.

This linear response justifies the use of an empirical formula for calculating the CPCP from interplanetary parameters. The CPCP is, of course, a major quantity governing the 
dynamics of the magnetosphere since it drives the plasma convection cycle. This empirical relation was obtained by Boyle, Reiff, and Hairston (1997) from a statistical analysis of several years of observations made by the DMSP satellites. It is given by

$$
\Phi_{\mathrm{A}}=10^{-4} V^{2}+11.7 B \sin ^{3}(\theta / 2)(\mathrm{kV}),
$$

where $V$ is in $\mathrm{km} \mathrm{s}^{-1}, B$ is in nT and angle $\theta=\arccos \left(B_{z} / B\right)$ with $B_{z}$ in GSM coordinates (i.e., the clock angle of the IMF). This potential gives a contribution to the CPCP, which is dependent on the solar wind parameters, i.e. the dayside source (as opposed to the nightside contribution, which is an independent source of convection and can give a significant contribution to the total CPCP during substorm expansion and recovery phases, with CPCP increases by a few tens of $\mathrm{kV}$ (Lockwood et al., 2009; Kullen et al., 2010; Gordeev et al., 2011). The first term depends only on the velocity of the solar wind, while the second term is based on the interplanetary magnetic field and its orientation in the GSM $Y Z$ plane.

The time sequence of the "Boyle" potential $\Phi_{\mathrm{A}}$ is plotted in Figure 11, panel 2. The average value is $\Phi_{B}=37.3 \pm 20.2 \mathrm{kV}$. At this high temporal resolution, isolated values approach $\sim 240 \mathrm{kV}$, which is typical of saturated CPCP (dependent on solar wind dynamic pressure and ionospheric conductivity; e.g. Siscoe et al., 2002; Hairston, Hill, and Heelis, 2003; Hairston, Drake, and Skoug, 2005). For comparison purposes, typical CPCP values lie in the range $[40, \sim 80] \mathrm{kV}$, and a CPCP $<40 \mathrm{kV}$ would correspond to quiet conditions [Marc Hairston, private communication, 2012]. Thus the bulk of our values lie in the lower half of this range.

The third panel of Figure 11 shows the bulk flows in 2008. As noted earlier, a succession of slow and fast streams is evident. In the bottom panel we plot the auroral AL electrojet index. One can see that there is practically a one-to-one correspondence between AL intensifications and CIRs/SIRs (see vertical red guidelines). This suggests that the part of the magnetosphere driving due to reconnection comes from the Alfvénic fluctuations in the fast streams of stream-stream interaction regions where $B_{z}$ fluctuates about $0 \mathrm{nT}$ (Tsurutani and Gonzalez, 1987).

We now give two examples in our data set of these Alfvénic fluctuations. Figure 12 shows the first example. For 29 September -8 October 2008 the panels give the proton density, temperature, the magnetic field strength and components, the velocity components and the auroral AE index. GSE coordinates are used. A very fast stream, reaching above $700 \mathrm{~km} \mathrm{~s}^{-1}$, is overtaking a slower stream. The bottom panel shows the auroral activity. We analyze further the interval 1-8 October, marked in the bottom panel by a horizontal bar. We form 13-point averages to represent the background magnetic field and velocity vectors. Subtracting this from the measurements gives us the perturbations. From these we determine the fluctuations perpendicular to the background magnetic field $\left(\Delta \mathbf{B}_{\perp}, \Delta \mathbf{V}_{\perp}\right)$ and check the Alfvén relation component-wise $\left.\Delta \mathbf{B}_{\perp}=\left(\mu_{0} \rho\right)^{1 / 2} \Delta \mathbf{V}_{\perp} \equiv \Delta \mathbf{A}_{\perp}\right)$, where $\rho$ is the mass density. The resulting scatter plots are shown in Figure 13. The straight lines are the regression lines. We find very good correlation between the components, valid to above the $99.9 \%$ confidence level. The slopes are $1.0(x), 0.8(y)$ and $1.0(z)$ for the various components. The positive correlation implies waves propagating against the field. From Figure 12 we see that average $B_{x}>0$ (average $=1.64 \mathrm{nT}$; sunward) so the waves are propagating antisunward.

A second example is that of 23-31 March 2009. Figures 14 and 15 show similar data to that of the previous example. The high-speed stream reached much lower values than in the first example. The five-day period 25-29 March is analyzed further. Here the average 
Figure 12 Data at one-minute resolution for the period 29 September -8 October 2008. From top to bottom: The proton density, temperature, magnetic field strength and components, the velocity components and the AE index. The horizontal bar in the last panel gives the eight-day interval examined for Alfvénic fluctuations.

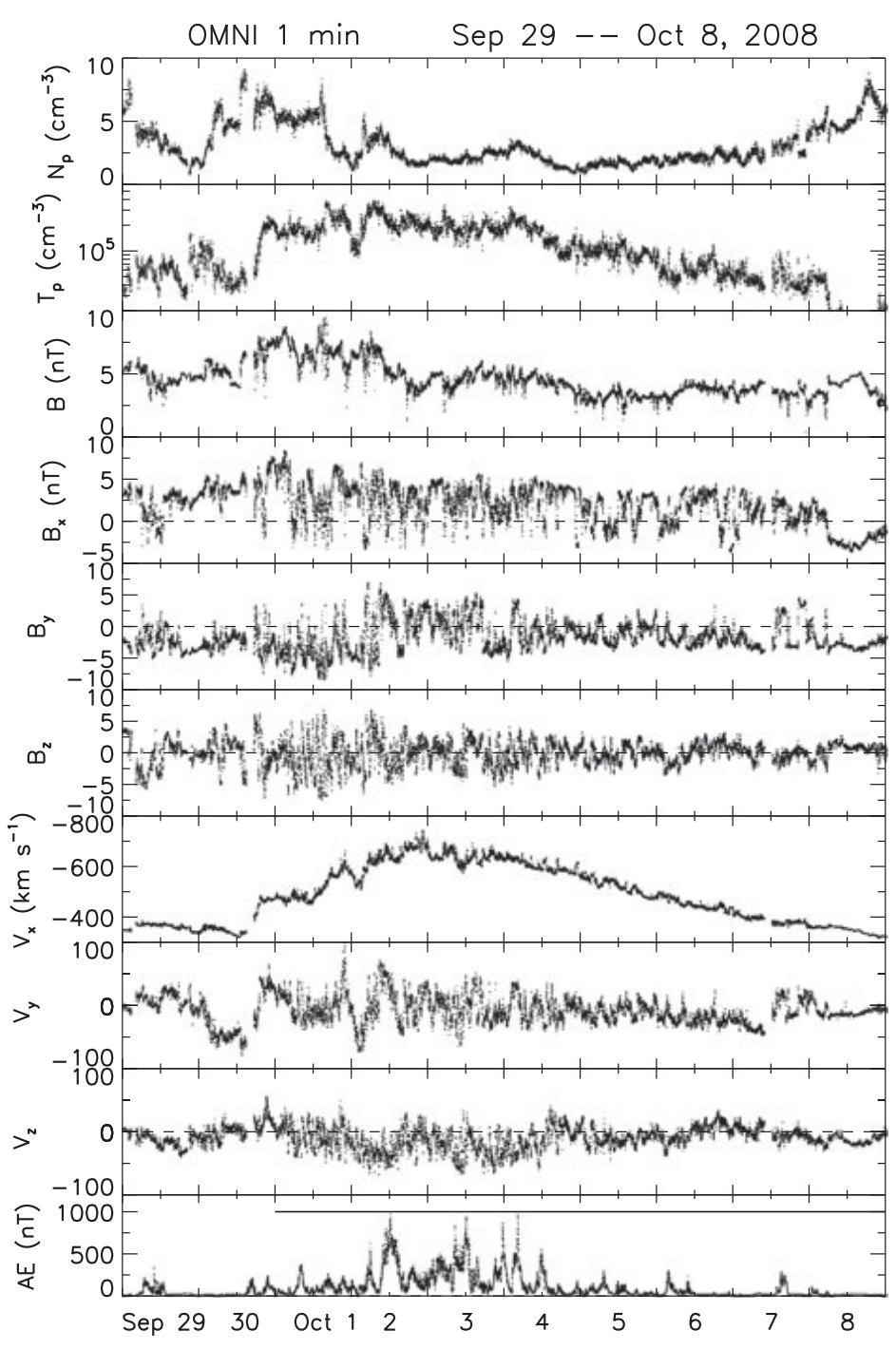

$B_{x}$ is $<0 \mathrm{nT}(-1.5 \mathrm{nT})$. For waves propagating antisunward, the field and flow components should be anticorrelated, consistent with Figure 15. Again, a reliable anti-correlation is found with slopes $-0.8(x),-0.8(y),-0.9(z)$.

\subsection{Shape and Location of Bow Shock and Magnetopause}

We do not propose to examine this topic over the entire 3-year period. To limit our scope, we consider Figure 16, which shows in the upper panel daily averages from the OMNI data base of the magnetic energy density and in the lower panel daily averages of the kinetic energy density. The plot is truncated from below to highlight a feature we are interested in, marked by the red arrows. During this about three-month period (27 March-26 June 2009), corresponding to CR 2081 (part) - 2084, we have a clear energy "hole" where both energy densities reach their lowest average values. The bottom panel shows the Alfvén Mach number over the three-year period.

To characterize the "energy hole" further, Figure 17 shows time profiles of select magnetic field and plasma parameters for the 91-day period of lowest $E_{\text {mag }}$ and $E_{\text {kin }}$. From top to bottom: the proton number density, bulk speed, total field strength, temperature, dynamic pressure and the Alfvén Mach number. The data are 5-min averages. Keeping in mind that 
Figure 13 The panels show the $x, y, z$ components of the magnetic field perturbations perpendicular to the background magnetic field plotted as a function of the corresponding velocity components multiplied by $\operatorname{sqrt}\left(\mu_{0} \rho\right)$, where $\rho$ is the mass density. The regression lines are shown. $R_{x}, y, z$ represent the respective correlation coefficients.
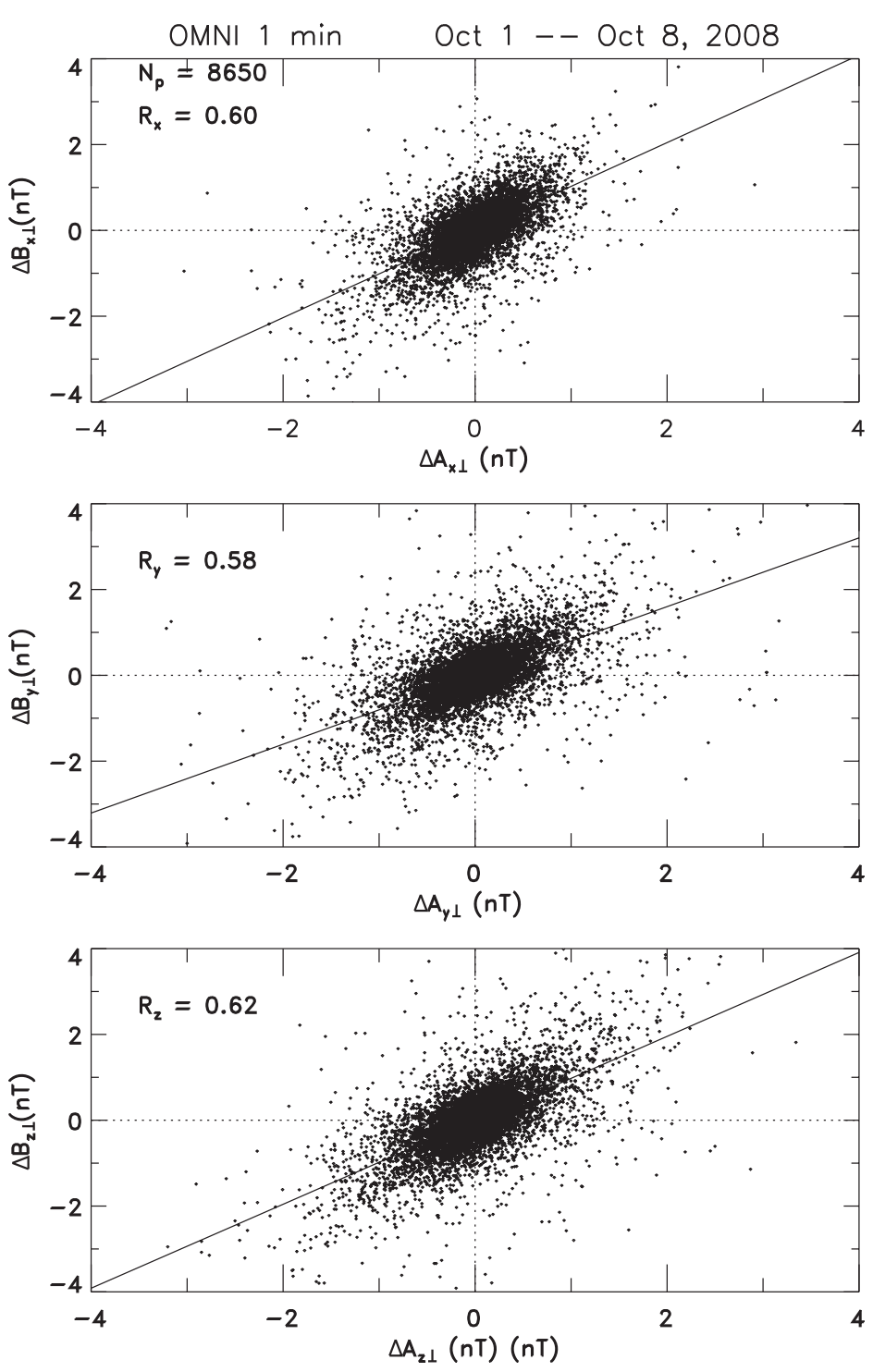

fast co-rotating streams are hot and the slow flows are cold, we have drawn vertical guidelines passing through (most) minima in $T$ in the first five panels (done by eye). These then typically occur at stream interfaces (SIs), where the plasma and field are compressed and the flow exhibits a sharp positive gradient. This is true even when a slow stream is overtaking a still slower stream. Some average values are: $N: 5.6 \mathrm{~cm}^{-3} ; P$ (dynamic pressure): $1.1 \mathrm{nPa}$ (about one-half of its historic average of $2.2 \mathrm{nPa}$ ); $B: 3.7 \mathrm{nT} ; M_{\mathrm{A}}: 12.0$, and $B_{z}$ (GSM): $-0.2 \pm 2.0 \mathrm{nT}$ (not shown). There is considerable fluctuation about these averages, of course, but the values show a solar wind of low kinetic pressure, weak magnetic field and small $B_{z}$ component. These features will determine the interaction with the terrestrial magnetosphere during the selected period that we discuss next. Also note the frequent SIRs/CIRs and in the later part we have slow-slower SIRs. We now ask: What is the effect of this on the shapes and location of the terrestrial magnetopause and bow shock?

To answer this, we proceeded as follows (see Harris, 2011): We compiled a data set of crossings of the magnetopause and bow shock made by Geotail, Cluster 1 and the THEMIS B and C spacecraft. These crossings cover both sides of the noon meridian. For Geotail we took plasma and magnetic field data from Low-Energy Particle (LEP) experiment (Mukai 
Figure 14 Similar to Figure 12, but for the period 23-29 March 2009.

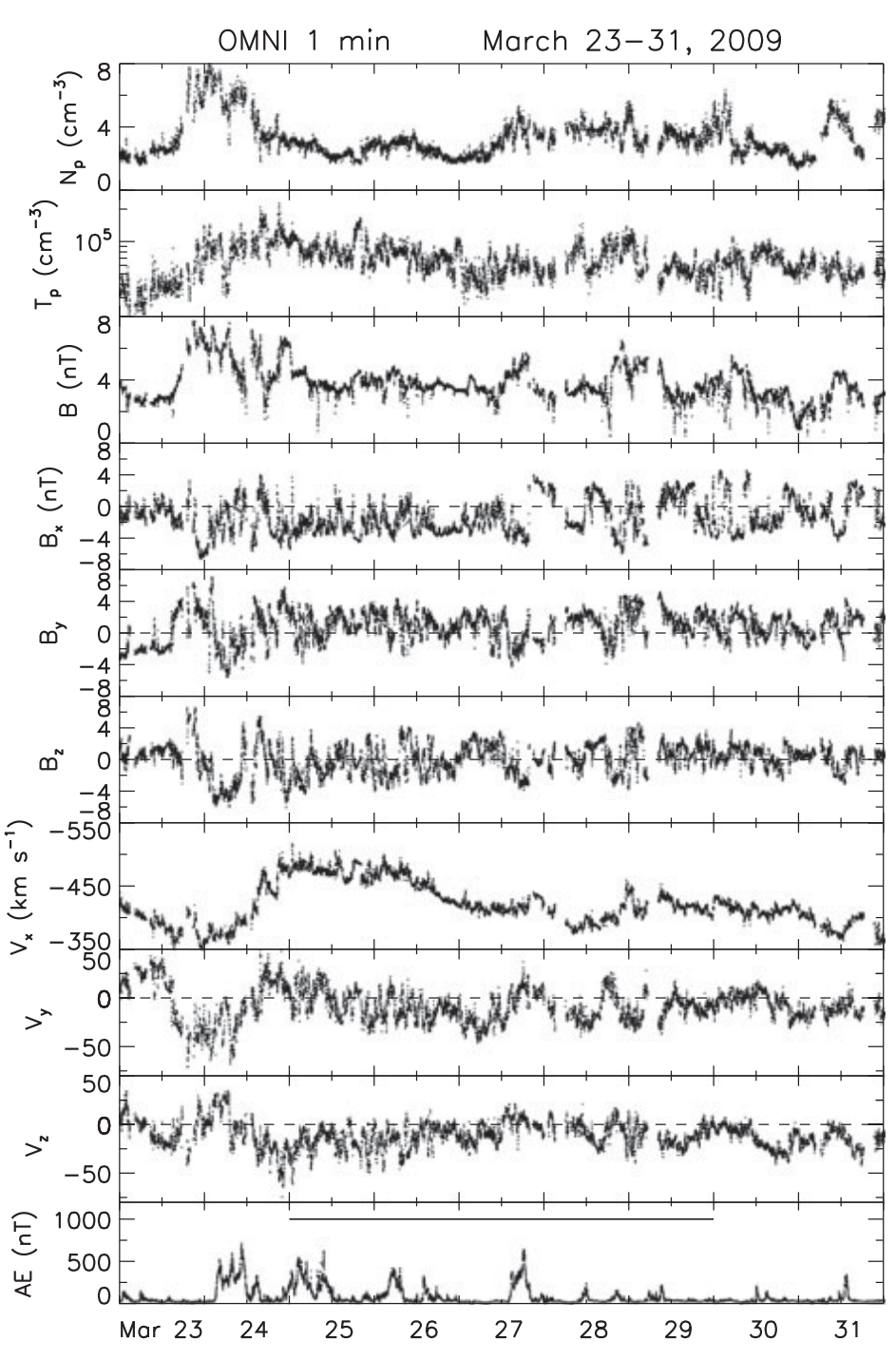

et al., 1994) and from the Geotail Magnetic Field experiment (Kokubun et al., 1995). For Cluster, we used data from the Cluster Ion Spectrometry (CIS) instrument (Rème et al., 2001) and from the Cluster Magnetic Field Investigation (Balogh et al., 2001). THEMIS data are from the Fluxgate Magnetometer (Auster et al., 2008) and from the Electrostatic Analyzer (ESA; McFadden et al., 2008). Interplanetary data were from the Magnetic Field Investigation (MFI, Lepping et al., 1995) and the Solar Wind Experiment (SWE, Ogilvie et al., 1995) on the Wind spacecraft. This search resulted in a total of 328 unambiguous magnetopause crossings and 271 bow shock crossings. The bulk of the data are in the X-range $[15,-20] R_{\mathrm{E}}$. The data were in GSE coordinates. We assumed a paraboloid (hyperboloid) of revolution for the magnetopause (bow shock), just as in Fairfield (1971). Each data point was then corrected for aberration due to the motion of the Earth around the Sun. To determine the solar wind conditions during this period, we used averages over the interplanetary parameters shown in Figure 17. When multiple magnetopause or bow shock crossings were observed within $30 \mathrm{~min}$, only the average magnetopause position is plotted. The interplanetary conditions correspond to this average time. Note that the three-month period under survey contained only one ICME (Richardson and Cane, 2010) and so the effect of ICMEs is not present in the result. 
Figure 15 Similar to Figure 13, but for the period 23-29 March 2009.
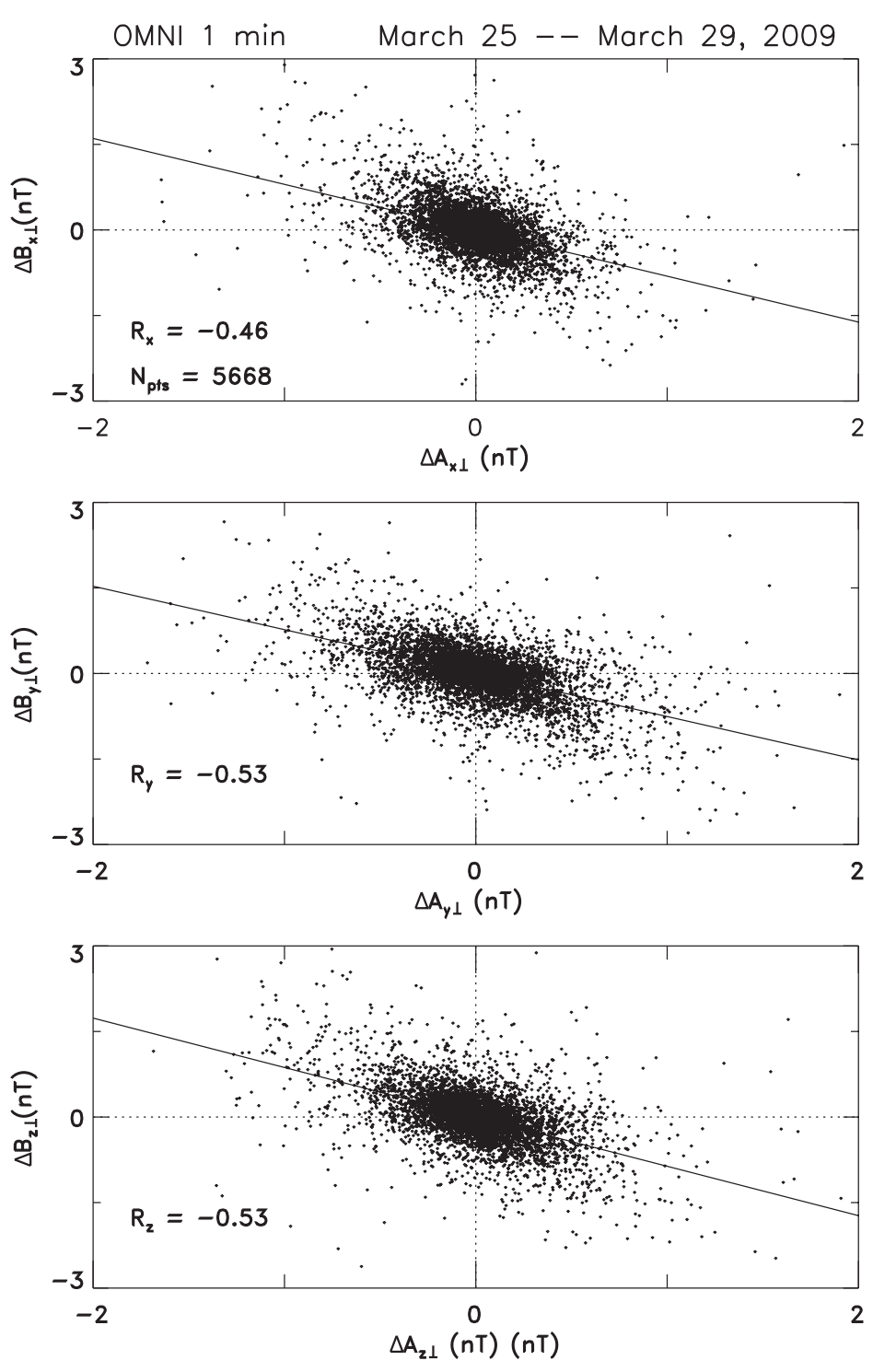

The IDL fitting routine AMOEBA is used. It computes a multi-dimensional minimization of a user-specified function. The expression we minimize is of the same functional form as that of Fairfield (1971) and is given by

$$
0=y^{2}+A x y+B x^{2}+C y+D x+E .
$$

Figure 18 shows in blue the resulting fit and in red the classic Fairfield (1971) result. The interplanetary data for Fairfield's model came from the IMP 1-6 spacecraft in the time frame (not continuous) from the end of 1963 to 1968, i.e. in the rising and maximum phase of Solar Cycle 20. The fitted coefficients are:

$$
a=-0.1261, \quad b=0.0245, \quad c=0.6717, \quad d=21.046, \quad e=-248.203 \text {. }
$$

Two interesting features may be noted: (1) The stand-off distance of $11.8 R_{\mathrm{E}}$ that we obtain is noticeably larger than that of Fairfield's result, and (2) and the magnetopause flares out more in $2007-2009$. Both are likely due to the low average $P_{\text {dyn }}$ during this three-month period.

In Figure 19 we compare the fitted magnetopause shape (in light blue) with two model magnetopauses, that of Shue et al. (1998; in red) and that of Sibeck, Lopez, Roelof (1991; 

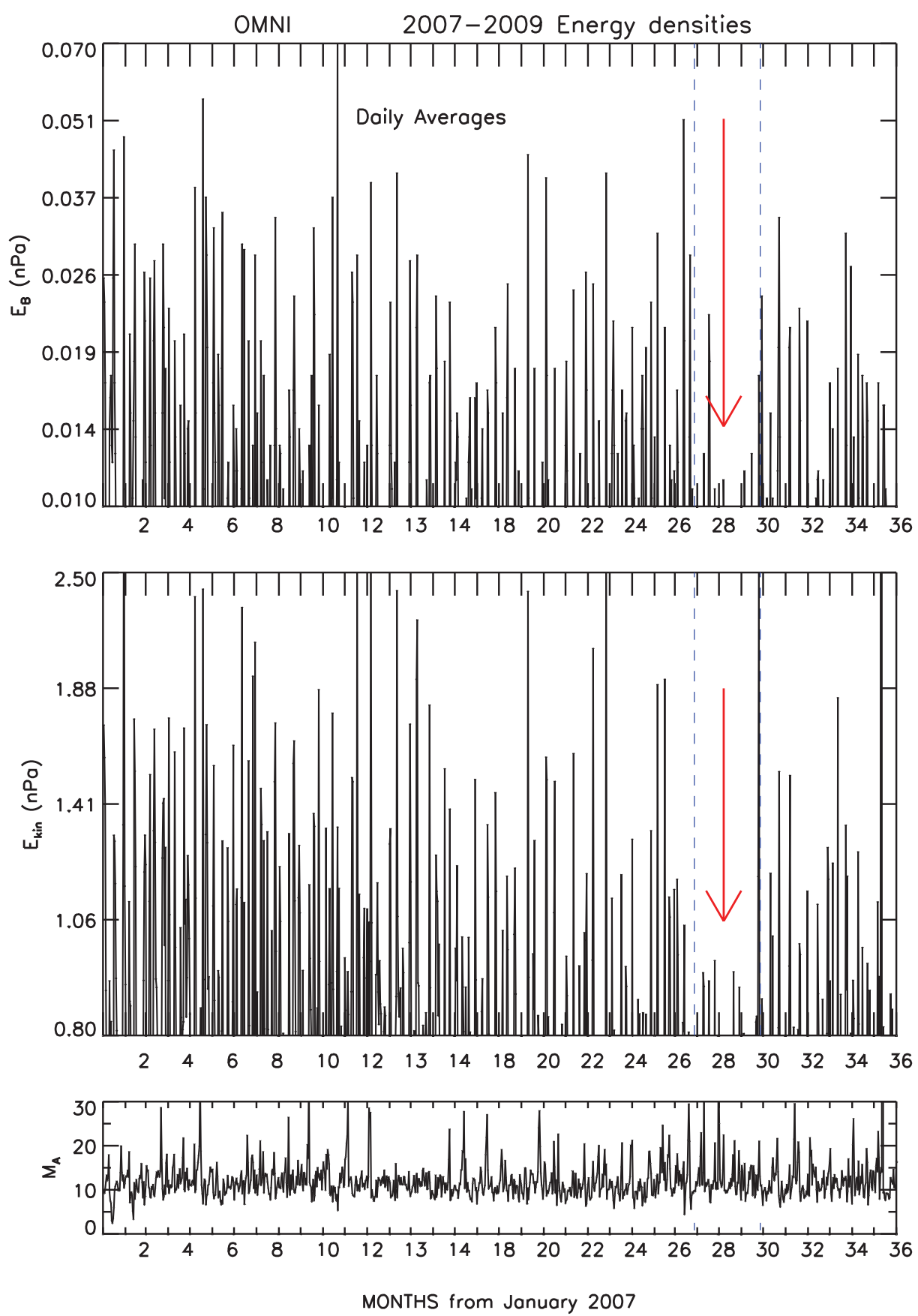

Figure 16 Daily averages of the magnetic and kinetic energy densities. Marked by arrows is a three-month period where both energy densities are on average the lowest reached in the three years under survey. The bottom panel shows the Alfvén Mach number over the three-year period.

in dark blue). The Sibeck, Lopez, Roelof model is based on 1821 crossings of the magnetopause. The data are binned by five values of the dynamic pressure. We plotted here the one corresponding to the average solar wind dynamic pressure in our chosen period.

In the Shue et al. (1998) work the influence of both solar $P_{\mathrm{dyn}}$ and IMF $B_{z}$ on the magnetopause shape are considered. No restriction is imposed on these parameters. Here we used the $B_{z}$-south version of Shue et al. (1998) since the average of this quantity - computed from 


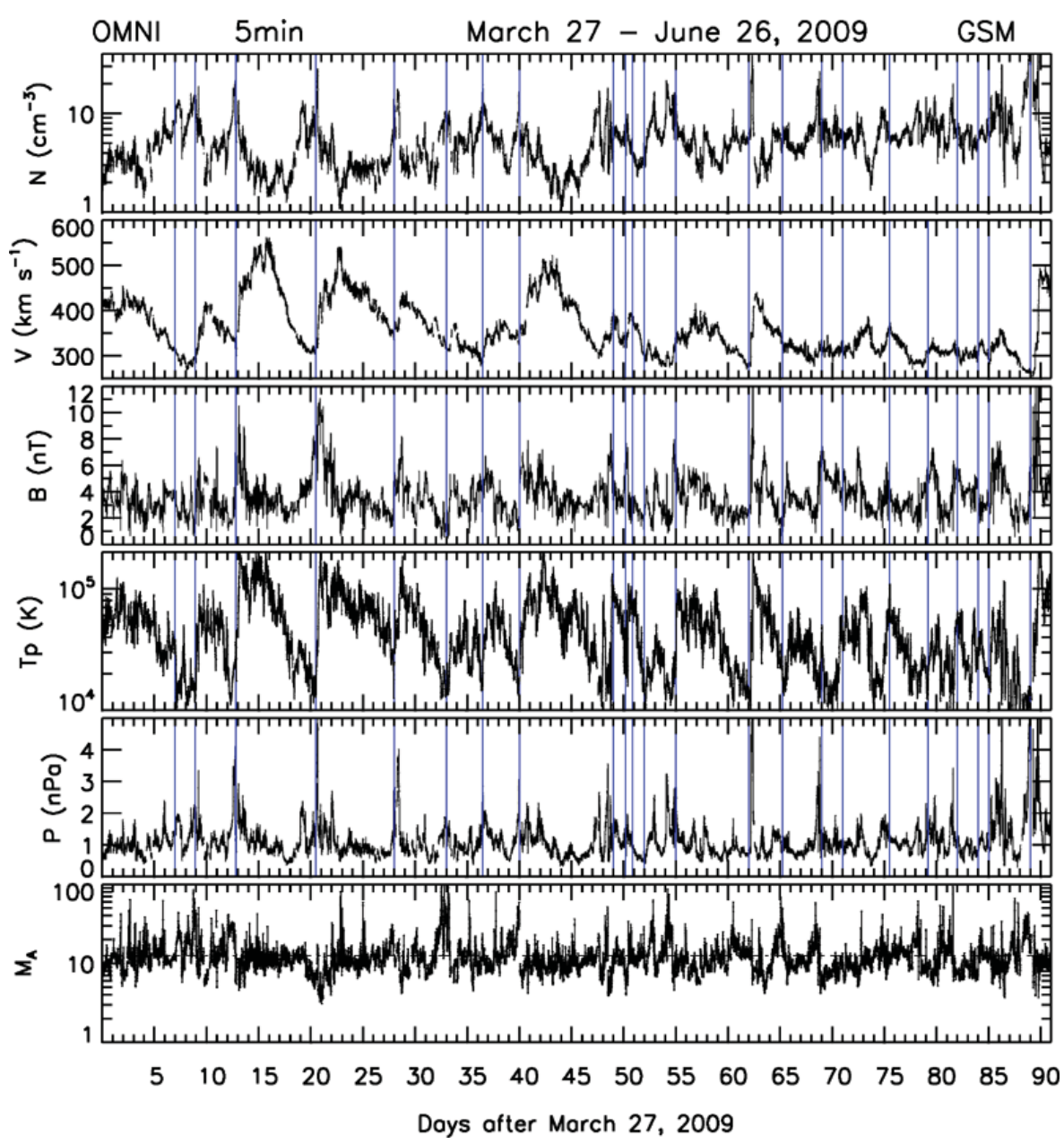

Figure 17 Time profiles of select plasma and magnetic field parameters: The proton number density, bulk speed, total field strength, temperature, dynamic pressure and Alfvén Mach number. Vertical blue guidelines are drawn through the minima in the temperature, $T$. For further details, see text.

daily values - in our period is slightly negative $(-0.2 \mathrm{nT})$. Shue et al. (1998) postulated a new functional form for the magnetopause shape, namely,

$$
r=r_{0}[2 /(1+\cos (\theta))]^{\alpha},
$$

where quantities $r_{0}$ and $\alpha$ are the stand-off distance and the tail flaring angle, respectively. Quantity $r$ gives the radial distance measured at an angle $\theta$ with the direction of $\mathbf{r}$.

The figure shows that both model magnetopauses underestimate the flaring of the magnetopause in our period. Of the two, Sibeck, Lopez, and Roelof (1991) model comes closer to reproducing the observed flaring in our three-month period. Both models underestimate the stand-off distance by $\sim 1 R_{\mathrm{E}}$.

The same three-month period in 2009 was employed to determine the bow shock shape and location. Figure 20 shows the resulting fit to the data (light blue trace). The fitted coefficients are:

$$
\begin{array}{ll}
a=0.0252, & b=0.4061, \quad c=-0.4576, \\
d=40.374, \quad e=-663.475 .
\end{array}
$$


Figure 18 The solar minimum magnetopause (blue curve) plotted in $(x, R)$ coordinates with $R=\left(X^{2}+Y^{2}\right)^{1 / 2}$. The red curve gives the empirical model of Fairfield (1971).

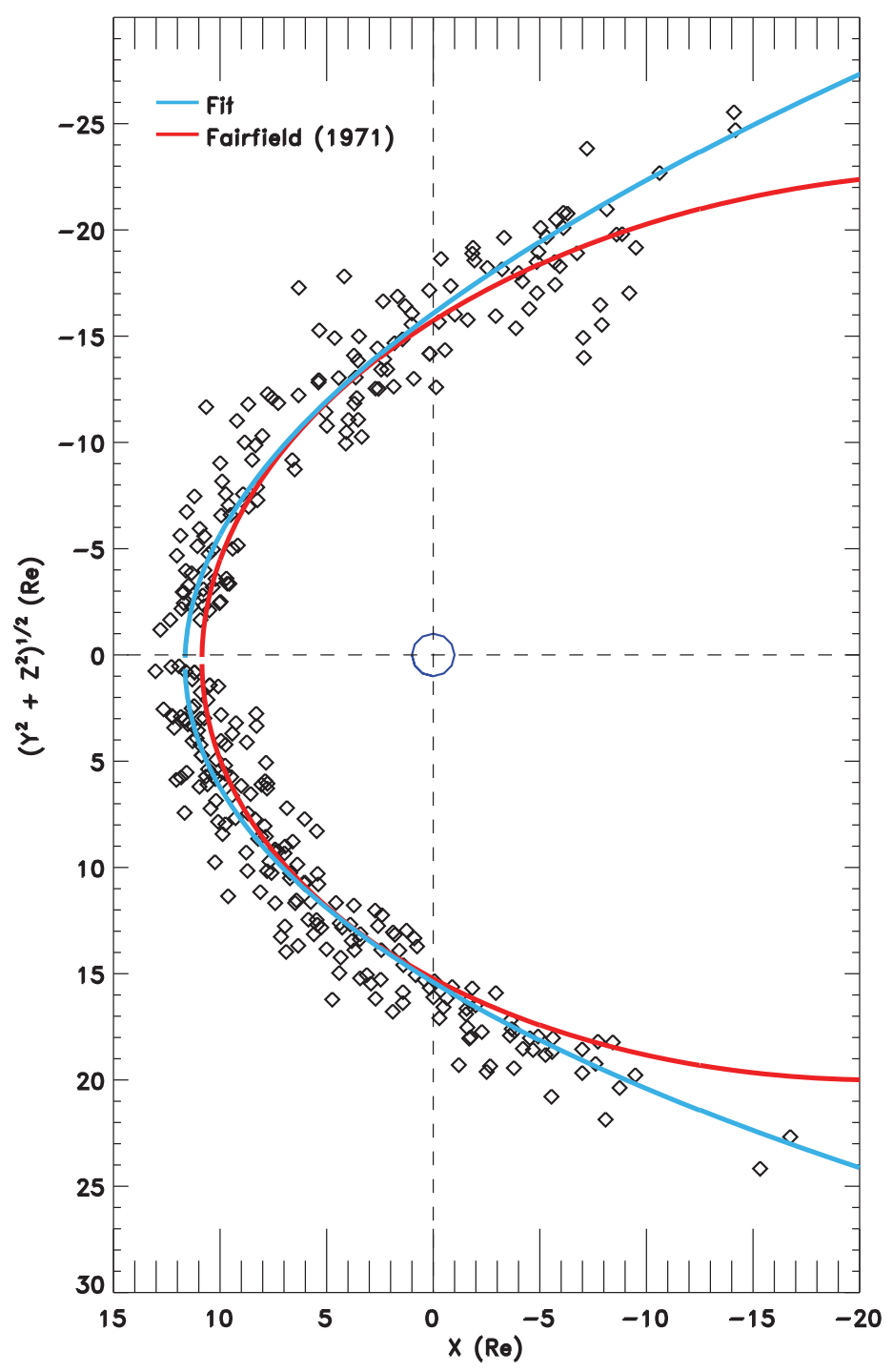

It is compared with Fairfield's 1971 model (red). The solar minimum bow shock is less flared than Fairfield's. Interestingly, the subsolar location (bow shock "nose") is practically the same. Two major factors affect the subsolar distance of the bow shock: When $M_{\mathrm{A}}$ increases, the bow shock moves earthward. At the same time, when the dynamic pressure decreases the magnetopause moves sunward and flares out. The stand-off distance of the bow shock is proportional to the object size (Farris and Russell, 1994), so that for lower $P_{\text {dyn }}$ the bow shock shifts sunward. It may be fortuitous that the two effects cancel each other, or that the data are not sufficient to show any remaining small offset.

In summary, the thinning by $1 R_{\mathrm{E}}$ of the subsolar magnetosheath during this three-month period is mainly due to a sunward motion of the magnetopause.

A small asymmetry is observed near the terminators of the bow shock. The dusk side crosses at $X=0$ at $26.5 R_{\mathrm{E}}$ while the dawn side crosses the terminator plane at $26.0 R_{\mathrm{E}}$. This asymmetry is small, however, and we shall not consider it further.

The nose of the minimized function describing the bow shock is at $14.35 R_{\mathrm{E}}$. With the result for the magnetopause, this corresponds to a magnetosheath thickness of $2.6 R_{\mathrm{E}}$. 
Figure 19 The solar minimum magnetopause (light blue trace) compared with the Sibeck, Lopez, Roelof (1991; dark blue) and the Shue et al. (1998; red) model magnetopauses. For calculating these model shapes the average solar minimum values for the dynamic pressure and $B_{z}$ were used.

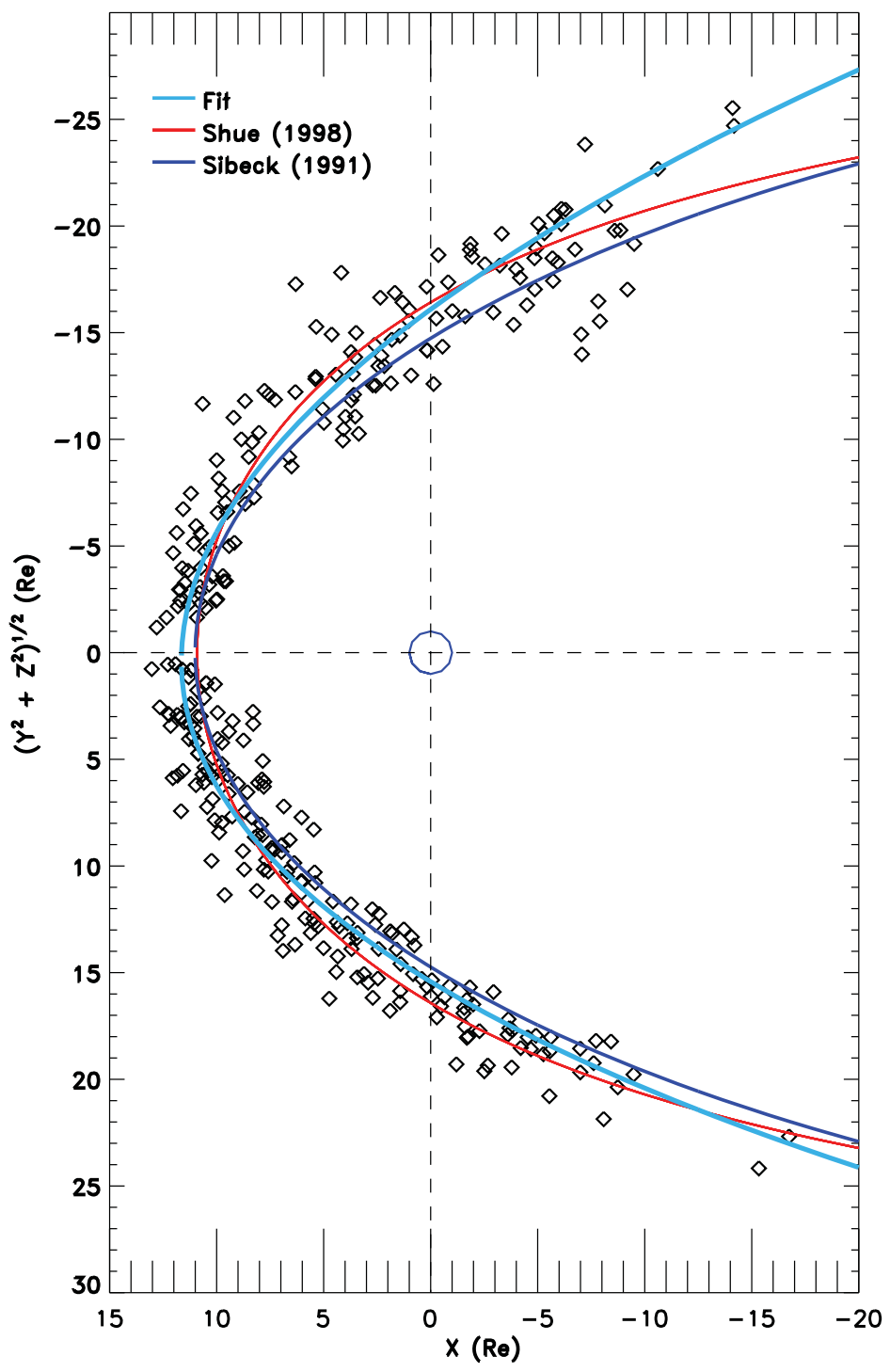

\section{Discussion and Conclusions}

We have discussed i) solar wind properties during the extreme solar activity minimum of $2007-2009$, and ii) two major aspects of the magnetospheric response. For the former we used data from ST-A and found that the density and magnetic field strength were, compared to other solar activity minima, significantly smaller. These values decreased the magnetic as well as the kinetic energy densities. The ratio of these energy densities, which is equal to the inverse square of the Alfvén Mach number, was unusually low. This has an effect on the interaction processes with the magnetosphere (see below). For ii), we first considered the solar wind electric field and found this to be small $(\sim 1.3 \mathrm{mV} / \mathrm{m})$. The implication was that the response of the magnetosphere, in so far as it depends on interplanetary parameters, remained linear (no saturation effects). This justified our use of an empirical formula - that of Boyle, Reiff, and Hairston (1997) - to find the average cross-polar cap potential from interplanetary parameters. The average value turned out to be $\approx 37 \mathrm{kV}$. Finally, we also derived empirical shapes of the magnetopause and bow shock using for these crossings on both sides of noon made by Cluster 1 (on the downside) and THEMIS B and C (on the duskside) during a three-month period when both solar wind energy densities reached 
Figure 20 Solar minimum bow shock (blue). In red is the Fairfield (1971) model bow shock.

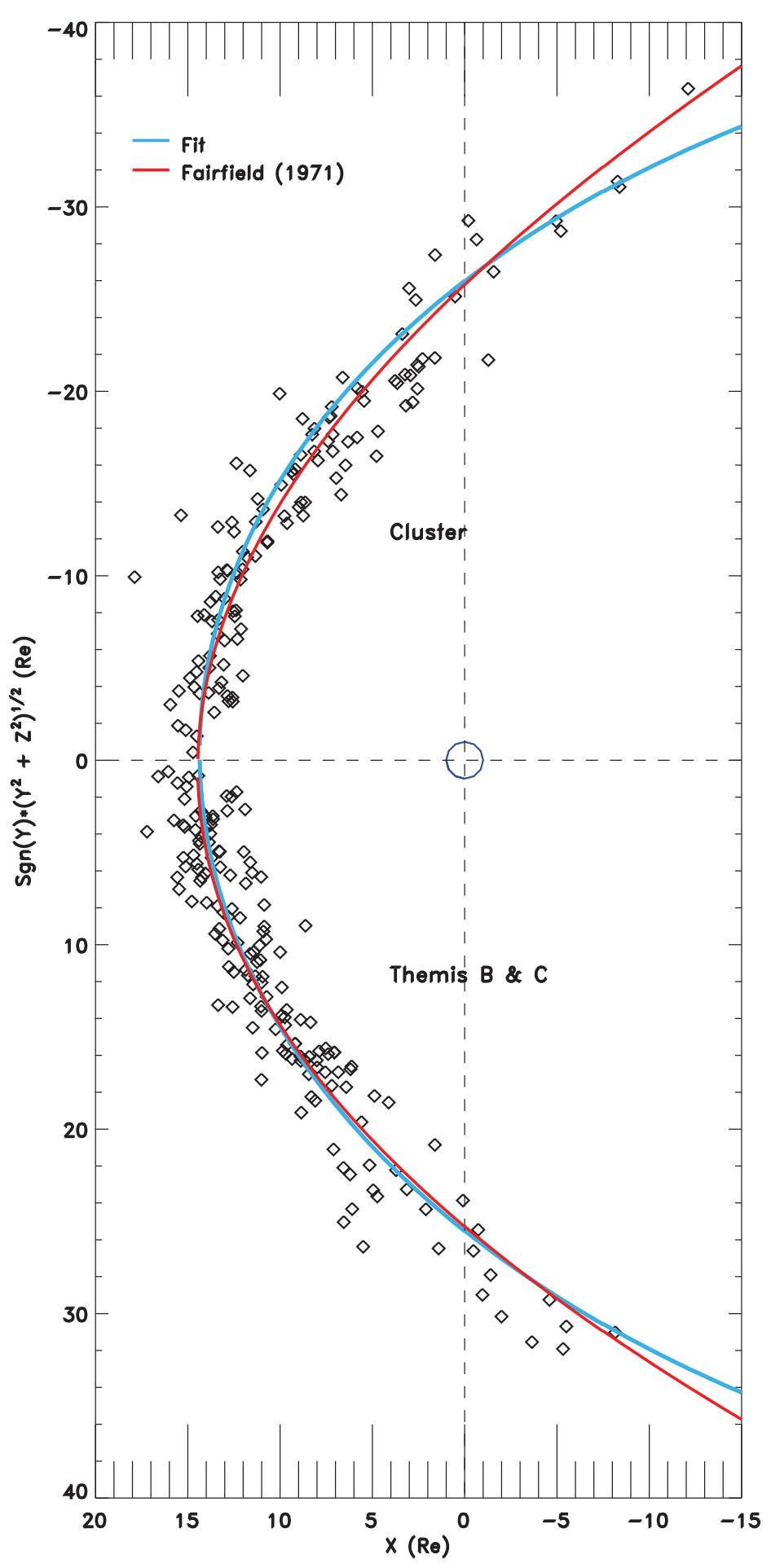

their lowest values in the three years under survey. These were augmented by Geotail observations. Comparing with Fairfield's (1971) classic result, there resulted a more flared out magnetopause whose stand-off distance (at $11.8 R_{\mathrm{E}}$ ) was larger by $\sim 1 R_{\mathrm{E}}$. The empirically determined bow shock was less flared out than Fairfield's. MHD effects tend to increase the flaring of the bow shock (see the Merka et al., 2003, their Figure 10). Thus our result is likely due to the fact that the solar wind approached closer to the gas dynamic limit (i.e. $M_{\mathrm{A}}$ was higher) than during Fairfield's period, which corresponded to the rising phase of Solar 
Cycle 20. The subsolar thickness of the magnetosheath was about $1 R_{\mathrm{E}}$ narrower than that of Fairfield. Assuming a normal thickness of $4 R_{\mathrm{E}}$, this amounts to a shrinkage of $\sim 25 \%$.

A major protagonist in this study is the solar wind (upstream) Alfvén Mach number, $M_{\mathrm{A}}$. The importance of this parameter is that the magnetic forces in the MHD momentum equation scale as $M_{\mathrm{A}}^{-2}$ (Erkaev, 1988; Farrugia et al., 1995). Thus increasing $M_{\mathrm{A}}$ leads to a magnetosheath in which magnetic forces are weaker. $\left(M_{\mathrm{A}}\right.$ equals infinity is the gas dynamic limit.) Typically at $1 \mathrm{AU}$, quantity $M_{\mathrm{A}}$ is $\approx 8-10$. An important exception noted in the literature is the low values of order $2-6$ achieved in magnetic clouds/ICMEs (Farrugia et al., 1995), which are magnetically dominated (low $\beta$ ) structures. This accounts for the unusual magnetosheath properties during their passage at Earth and a concomitant altered interaction with the magnetosphere (e.g. Lavraud and Borovsky, 2008). During the long minimum we are investigating, $M_{\mathrm{A}}$ often reached very high values with an average of 12.9 (see $e$.g. bottom panel of Figure 16). Thus magnetic forces are weak in the solar wind prevailing in these three years. This, coupled with the repetitive high speeds reached in what is almost a continued stream-stream interaction process, provide favorable conditions for the Kelvin-Helmholtz instability to grow, since (a) they weaken the stabilizing magnetic tension forces, and (b) enhance a destabilizing factor (the velocity shear) a good fraction of the time, when highspeed flows were prominent. Indeed Engebretson et al. (1998), studying the effect of two repetitive co-rotating stream interactions over a six-month period, found that auroral UltraLow Frequency (ULF) wave power in the PC 5 range had a power law dependence on solar wind velocity. The ULF power was strongest at the leading edge of high-speed streams. They obtained good agreement with a numerical simulation which assumed that the KelvinHelmholtz (KH) instability was mediating this activity. Arguing by analogy, we hypothesize that the $\mathrm{KH}$ instability, one of the contributors to viscous-type solar wind-magnetosphere interactions, played a major role in transferring solar wind energy and momentum to the magnetosphere during these three years.

We note that in 2007-2009 the ICME occurrence rate at 1 AU was low in comparison to the previous minimum, and the few ICMEs that were detected had lower field strengths and lower speeds (Lepping et al., 2011). In early 2007, less than one ICME per month was observed, though this number increased somewhat at the end of 2008 presumably due to deflections of CMEs towards the ecliptic (Kilpua et al., 2009). Due to the low CME/ICME speeds, about half of the ICMEs were swept up by CIRs before reaching $1 \mathrm{AU}$ in 2007 and early 2008 (Kilpua et al., 2009; further ICME identifications are also provided by Richardson and Cane (2010)). Thus, during 2007-2009 the interaction of the solar wind with the magnetosphere was clearly not dominated by ICMEs but by CIRs/SIRs.

In fact, as already mentioned, the interplanetary medium resembled a continued streamstream interaction process. The Alfvén waves in the high-speed streams propagating outward from the Sun are known to be the cause for continued auroral activity, which we also observe (Figure 11, bottom panel, and Figures 12, 14). As Tsurutani and Gonzalez (1987) pointed out, the $B_{z}$-fluctuations about zero in the high-speed streams are likely to cause reconnection and give rise to this activity ("HILDCAAS", short for high-intensity, longduration, continuous AE activity). So this reconnection interaction, too, contributed to the CPCP.

In Section 2.2 we also gave results on the energy budget in the interplanetary medium, discussing in particular quantity QI, which is the ratio of the magnetic-to-kinetic energy densities. We can put our results on this in a broader context. The values of QI obtained here can be compared with two cases studied in the literature: i) QIs in normal solar wind at $1 \mathrm{AU}$, and ii) QIs in interplanetary coronal mass ejections, which are magnetically dominated structures (low proton $\beta$ ). For i) we consider Osherovich, Fainberg, and Stone (1999) who gave 
a formula for QI in terms of SSN. This is QI $=a+b \cdot \operatorname{SSN}\left(a=7.6 \times 10^{-3} \pm 0.15 \times 10^{-3}\right.$; $\left.b=7.8 \times 10^{-5} \pm 0.3 \times 10^{-5}\right)$. Using SSN numbers for $2007-2009$ from the NOAA/National Geophysical Data Center (NGDC) website, we obtain an average value of QI $=0.007$, in good agreement with our data, particularly for 2007-2008 (Figure 8).

In a series of papers, (Leitner et al., 2009; Leitner, Vörös, and Leubner, 2009; Leitner and Farrugia, 2010; Leitner, Farrugia, and Vörös, 2011) discussed other aspects of the probability distribution functions of QI, which complement the present work and even extend it in the detailed analysis of the shape of the distribution functions. They also gave other details, such as the distributions of QI in the slow and fast solar winds, separately. These are beyond the scope of our work here, but the reader is encouraged to look at these papers for more detail.

Acknowledgements We are grateful to the referee for many helpful suggestions. We thank Marc Hairston, Jana Safrankova and Zdenek Nemecek for helpful comments. For the data used in the geomagnetic response section, we thank the World Data Center for Geomagnetism at the Kyoto web site, Japan; the OMNI website at Goddard Space Flight Center, USA; and the Defense Meteorological Satellite Program (DMSP) program, NOAA. Work at UNH was supported by NASA grants NNX10AQ29G and NAS5-03131. This work has received funding from the European Commissions's Seventh Framework Programme (FP7/2007 - 2013) under the grant agreement No. 263253 [COMESEP]. V.O. was supported by NASA Grant NNXO9AR80G. M.T. greatly acknowledges the Austrian Science Fund (FWF): FWF V195-N16. This research was supported by a Marie Curie International Outgoing Fellowship within the 7th European Community Framework Program.

\section{References}

Auster, H.U., Glassmeier, K.H., Magnes, W., Aydogar, O., Baumjohann, W., Constantinescu, D., Fischer, D., et al.: 2008, The THEMIS fluxgate magnetometer. Space Sci. Rev. 141, 235.

Balogh, A., Carr, C.M., Acũna, M.H., Dunlop, M.W., Beek, T.J., Brown, P., Fornacon, K.-H., et al.: 2001, The cluster magnetic field investigation: overview of in-flight performance and initial results. Ann. Geophys. 19, 1207.

Boyle, C.B., Reiff, P.H., Hairston, M.R.: 1997, Empirical polar cap potentials. J. Geophys. Res. 102, 111.

Burlaga, L.F., Lazarus, A.J.: 2000, Log-normal distributions and spectra of solar wind plasma fluctuations: Wind 1995 - 1998. J. Geophys. Res. 105, 2357.

Clette, F., Berghmans, D., Vanlommel, P.R., van der Linden, A.M., Koeckelenbergh, A., Wauters, L.: 2007, From the Wolf number to the international sunspot index: 25 years of SIDC. Adv. Space Res. 40, 919.

Cranmer, S.R., Hoeksema, J.T., Kohl, J.L. (eds.): 2010, SOHO-23: Understanding a Peculiar Solar Minimum. ASP CS-428.

Engebretson, M., Glassmeier, K.-H., Stellmacher, M., Hughes, E.J., Lühr, H.: 1998, The dependence of highlatitude PC5 wave power on solar wind velocity and on the phase of high-speed solar wind streams. J. Geophys. Res. 103, 26271.

Erkaev, N.V.: 1988, Results of the investigation of MHD flow around the magnetosphere. Geomagn. Aeron. 28, 455.

Fainberg, J., Osherovich, V.A.: 2002, Solar wind quasi-invariant as a heliospheric index of solar activity. In: Solar Variability: From Core to Outer Frontiers SP-506, ESA, Noordwijk, 43.

Fainberg, J., Osherovich, V.A., Stone, R.G.: 2001, Pioneer Venus Orbiter observations of a solar wind quasiinvariant. Geophys. Res. Lett. 28, 1447. doi:10.1029/2000GL012160.

Fairfield, D.H.: 1971, Average and unusual locations of the Earth's magnetopause and bow shock. J. Geophys. Res. 76, 6700.

Farris, M.H., Russell, C.T.: 1994, Determining the standoff distance of the bow shock: Mach number dependence and use of models. J. Geophys. Res. 99, 17681.

Farrugia, C.J., Erkaev, N.V., Biernat, H.K., Burlaga, L.F.: 1995, Anomalous magnetosheath properties during Earth passage of an interplanetary magnetic cloud. J. Geophys. Res. 100, 19245.

Feynman, J., Ruzmaikin, A.: 2011, The Sun's strange behavior: Maunder Minimum or Gleissberg Cycle? Solar Phys. 272, 351.

Galvin, A.B., Kistler, L.M., Popecki, M.A., Farrugia, C.J., Simunac, K.D.C., Ellis, E., Möbius, E., et al.: 2008, The Plasma and Suprathermal Ion Composition (PLASTIC) investigation on the STEREO observatories. Space Sci. Rev. 136, 437. doi:10.1007/s11214-007-9296-x. 
Gordeev, E.I., Sergeev, V., Pulkkinen, T.I., Palmroth, M.: 2011, Contribution of magnetotail reconnection to the cross-polar cap electric potential drop. J. Geophys. Res. 116, A08219. doi:10.1029/2011JA016609.

Gosling, J.T., Pizzo, V.J.: 1999, Formation and evolution of corotating interaction regions and their three dimensional structure. Space Sci. Rev. 89, 21.

Hairston, M.R., Hill, T.W., Heelis, R.A.: 2003, Observed saturation of the ionospheric polar cap potential during the 31 March 2001 storm. Geophys. Res. Lett. 30, 1325. doi:10.1029/2002GL015894.

Hairston, M.R., Drake, T.R., Skoug, R.: 2005, Saturation of the ionospheric polar cap potential during the October-November, 2003 superstorms. J. Geophys. Res. 110, A09S26. doi:10.1029/2004JA010864.

Harris, B.: 2011, Observational aspects of IMF draping around the magnetosphere. MS Thesis, UNH.

Kan, J.R., Lee, L.C.: 1979, Energy coupling function and solar wind-magnetosphere dynamo. Geophys. Res. Lett. 6, 577.

Kilpua, E.K.J., Pomoell, J., Voulidas, A., Vainio, R., Luhmann, J., Li, Y., Schroeder, P., Galvin, A.B., Simunac, K.: 2009, Stereo observations of interplanetary coronal mass ejections during solar minimum period. Ann. Geophys. 27, 4491.

King, J.H., Papitashvili, N.E.: 2005, Solar wind spatial scales in and comparisons of hourly Wind and ACE plasma and magnetic field data. J. Geophys. Res. 110, A02104. doi:10.1029/2004JA010649.

Kokubun, S., Yamamoto, T., Acũna, M.H., Hayashi, K., Shiokawa, K., Kawano, H.: 1995, The geotail magnetic field experiment. J. Geomagn. Geoelectr. 46, 7.

Kullen, A., Karlsson, T., Cumnock, J.A., Sundberg, T.: 2010, Occurrence and properties of substorms associated with pseudobreakups. J. Geophys. Res. 114, 115, A12310. doi:10.1029/2010JA015866.

Lavraud, B., Borovsky, J.E.: 2008, Altered solar wind-magnetosphere interaction at low Mach numbers: Coronal mass ejections. J. Geophys. Res. 114, A00B08. doi:10.0129/2008JA13192.

Leitner, M., Farrugia, C.J.: 2010, Solar wind quasi-invariant within ICMEs. In: Solar Wind 12, AIP CP-1216, 652.

Leitner, M., Vörös, Z., Leubner, M.P.: 2009, Introducing log-kappa distributions for solar wind analysis. J. Geophys. Res. 114, A12104. doi:10.1029/2009JA014476.

Leitner, M., Farrugia, C.J., Vörös, Z.: 2011, Change of solar wind quasi-invariant in solar cycle 23 -analysis of PDFs. J. Atmos. Solar-Terr. Phys. 73, 290.

Leitner, M., Farrugia, C.J., Osherovich, V.A., Fainberg, J., Biernat, H.K., Ogilvie, K.W., Schwenn, R., Torbert, R.B.: 2005, The relative distribution of the magnetic and plasma kinetic energy densities in the inner heliosphere ( $<1 \mathrm{AU})$. In: Solar Wind 11/SOHO 16, Connecting Sun and Heliosphere SP-592, ESA, Noordwijk, 743.

Leitner, M., Farrugia, C.J., Galvin, A., Biernat, H.K., Osherovich, V.A.: 2009, The solar wind quasi-invariant observed by STEREO-A and B at solar minimum 2007, and comparison with two other minima. Solar Phys. 259, 381. doi:10.1007/s11207-009-9412-z.

Lepping, R.P., Acũna, M.H., Burlaga, L.F., Farrell, W.M., Slavin, J.A., Schatten, K.H., Mariani, F., et al.: 1995, The wind magnetic field investigation. Space Sci. Rev. 71, 207.

Lepping, R.P., Wu, C.C., Berdichevsky, D.B., Szabo, A.: 2011, Magnetic clouds at/near the $2007-$ 2009 minimum: Frequency of occurrence and some unusual properties. Solar Phys. 274. doi:10.1007/s11207-010-9646-9.

Lockwood, M., Hairston, M., Finch, I., Rouillard, A.: 2009, Transpolar voltage and polar cap UX during the substorm cycle. J. Geophys. Res. 114, A0120. doi:10.1029/2008JA013697.

Luhmann, J., Zhang, T.L., Petrinec, S., Russell, C., Gazis, P., Barnes, A.: 1993, Solar cycle 21 effects on the interplanetary magnetic field and related parameters at 0.7 and 1.0 AU. J. Geophys. Res. 98, 5559.

Luhmann, J.G., Curtis, D.W., Schroeder, P., McCauley, J., Lin, R.P., Larson, D.E., Bale, S.D., et al.: 2008, STEREO IMPACT investigation goals, measurements, and data products. Space Sci. Rev. 136, 117.

McFadden, J.P., Carlson, C.W., Larson, D., Ludlam, M., Abiad, R., Elliott, B., Turin, P., et al.: 2008, The THEMIS ESA plasma instrument and in-flight calibration. Space Sci. Rev. 141, 277.

Merka, J., Szabo, A., Safrankova, J., Nemecek, S.: 2003, Earth's bow shock and magnetopause in the case of a field-aligned upstream flow: Observation and model comparison. J. Geophys. Res. 108, 1269. doi:10.1029/2002JA009697.

Mukai, T., Machida, S., Hirahara, M., Teresawa, T., Kaya, N., Obara, T., Ejiri, M., Nishida, A.: 1994, The Low-Energy Particle (LEP) experiment onboard the Geotail satellite. J. Geomagn. Geoelectr. 46, 669.

Mühlbachler, S., Farrugia, C.J., Raeder, J., Biernat, H.K., Torbert, R.B.: 2005, A statistical investigation of dayside magnetosphere erosion showing saturation of response. J. Geophys. Res. 110, A11207. doi:10.1029/2005JA011177.

Ogilvie, K.W., Chornay, D.J., Fritzenreiter, R.J., Hunsaker, F., Keller, J., Lobell, J., Miller, G., et al.: 1995 , SWE, A comprehensive plasma instrument for the Wind spacecraft. Space Sci. Rev. 71, 55.

Osherovich, V.A., Fainberg, J., Stone, R.G.: 1999, Solar wind quasi-invariant as a new index of solar activity. Geophys. Res. Lett. 26, 2597. doi:10.1029/1999GL90058. 
Rème, H., Aoustin, C., Bosqued, J.M., Dandouras, I., Lavraud, B., Sauvaud, J.A., Barthe, A., et al.: 2001, First multi-spacecraft ion measurements in and near the Earth's magnetosphere with the identical Cluster Ion Spectrometry (CIS) experiment. Ann. Geophys. 19, 1303.

Richardson, I.G., Cane, H.V.: 2010, Near-Earth interplanetary coronal mass ejections during solar cycle 23 (1996-2009): Catalog and summary of properties. Solar Phys. 264, 189.

Scherrer, P.H., Wilcox, J.M., Svalgaard, L., Duvall, T.L. Jr., Dittmer, P.H., Gustafson, E.K.: 1977, The mean magnetic field of the Sun: Observations at Stanford. Solar Phys. 54, 353.

Shue, J.-H., Song, P., Russell, C.T., Steinberg, J.T., Chao, J.K., Zastenker, G., Vaisberg, O.L., et al.: 1998, Magnetopause location under extreme solar wind conditions. J. Geophys. Res. 103, 17691.

Sibeck, D.J., Lopez, R.E., Roelof, E.C.: 1991, Solar wind control of the magnetopause shape, location and motion. J. Geophys. Res. 96, 5489.

Siscoe, G.L., Erickson, E.M., Sonnerup, B.U.Ö., Maynard, N.C., Schoendorf, J.A., Siebert, K.D., Weimer, D.R., White, W.W., Wilson, G.R.: 2002, Hill model of transpolar potential saturation: Comparisons with MHD simulations. J. Geophys. Res. 107, 1075. doi:10.1029/2001JA000109.

Slavin, J.A., Holzer, R.E.: 1981, Solar wind flow about the terrestrial planets, 1. Modeling bow shock position and shape. J. Geophys. Res. 86, 11,401. doi:10.1029/JA086iA13.

Sonnerup, B.U.Ö.: 1974, Magnetopause reconnection rate. J. Geophys. Res. 79, 1546.

Tsurutani, B.T., Gonzalez, W.D.: 1987, The cause of high-intensity, long-duration, continuous AE activity (HILDCAAS): Interplanetary Alfvén wave trains. Planet. Space Sci. 35, 405.

Wang, Y.-M.: 2009, Coronal holes and open magnetic flux. Space Sci. Rev. 144, 383.

Wang, Y.-M., Robbrecht, E., Rouillard, A.P., Sheeley, N.R. Jr., Thernisien, A.F.R.: 2010, Formation and evolution of coronal holes following the emergence of active regions. Astrophys. J. 715, 39. 